ARTICLE

\title{
Cenpj/CPAP regulates progenitor divisions and neuronal migration in the cerebral cortex downstream of Ascl1
}

\author{
Patricia P. Garcez ${ }^{1}$, Javier Diaz-Alonso², Ivan Crespo-Enriquez ${ }^{3}$, Diogo Castro ${ }^{1, \dagger}$, Donald Bell ${ }^{4}$ \\ \& François Guillemot ${ }^{1}$
}

The proneural factor Ascl1 controls multiple steps of neurogenesis in the embryonic brain, including progenitor division and neuronal migration. Here we show that Cenpj, also known as CPAP, a microcephaly gene, is a transcriptional target of Ascl1 in the embryonic cerebral cortex. We have characterized the role of Cenpj during cortical development by in utero electroporation knockdown and found that silencing Cenpj in the ventricular zone disrupts centrosome biogenesis and randomizes the cleavage plane orientation of radial glia progenitors. Moreover, we show that downregulation of Cenpj in post-mitotic neurons increases stable microtubules and leads to slower neuronal migration, abnormal centrosome position and aberrant neuronal morphology. Moreover, rescue experiments shows that Cenpj mediates the role of Ascl1 in centrosome biogenesis in progenitor cells and in microtubule dynamics in migrating neurons. These data provide insights into genetic pathways controlling cortical development and primary microcephaly observed in humans with mutations in Cenpj.

\footnotetext{
${ }^{1}$ Division of Molecular Neurobiology, MRC National Institute for Medical Research, Mill Hill, London NW7 1AA, UK. ${ }^{2}$ Department of Biochemistry and Molecular Biology I, School of Biology and Instituto Universitario de Investigaciones Neuroquímicas (IUIN), Complutense University, 28040 Madrid, Spain. ${ }^{3}$ Department of Craniofacial Development \& Stem Cell Biology, King's College London, Guy's Tower Wing, Floor 27, London SE1 9RT, UK. ${ }^{4}$ Confocal and Image Analysis Laboratory, MRC National Institute for Medical Research, Mill Hill, London NW7 1AA, UK. †Present address: Instituto Gulbenkian de Ciência, Rua Quinta Grande 6, 2780-156 Oeiras, Portugal. Correspondence and requests for materials should be addressed to P.P.G. (email: ppgarcez@gmail.com) or to F.G. (email: fguille@nimr.mrc.ac.uk).
} 
$\mathrm{F}$ or the cerebral cortex to exert its crucial functions in cognition in humans, the different stages of its development, including the proliferation of its progenitors and the migration of its neurons, must proceed unencumbered. Defects in any of those steps can result in severe disorders, including microcephaly, lissencephaly or microlissencephaly ${ }^{1,2}$. Investigation of the mechanisms that control the neurogenesis thus contributes to our understanding of these severe neuropathologies.

The proneural transcriptional factor Ascl1/Mash1 is an important regulator of neurogenesis in different regions of the embryonic nervous system ${ }^{3,4}$. In the developing cerebral cortex, Ascll regulates both early and late aspects of neurogenesis, including the division of radial glia progenitors ${ }^{5}$ and the radial migration of post-mitotic neurons ${ }^{6,7}$. Ascl1 achieves its diverse functions through direct transcriptional regulation of a large number of target genes. These include effector molecules controlling particular aspects of the neurogenic process, such as cell cycle progression or the reorganization of the cytoskeleton associated with neuronal migration ${ }^{5,6,8}$. Here we have identified the centrosome-associated protein Cenpj (centromere protein $\mathrm{j}$, also known as CPAP) as a new and essential regulator of several crucial steps in the neurogenesis programme driven by Ascll.

Mutations in the human Cenpj gene are responsible for primary autosomal microcephalies, including Seckel syndrome, characterized by severely reduced brain sizes ${ }^{9-12}$. Downregulation of Cenpj in HeLa cells causes centrosome duplication defects that lead to spindle malformation and modifies the orientation of the cleavage plane ${ }^{13,14}$. In mice, Cenpj deficiency produces a Seckel syndrome-like phenotype with a twofold smaller head ${ }^{15}$. Loss of Cenpj function in mouse fibroblasts results in centrosome defects causing mitotic spindle malformation and cell cycle arrest in $\mathrm{G} 2 / \mathrm{M}$, as well as genomic instability ${ }^{15}$.

In this study, we have examined the contribution of Cenpj to cortical neurogenesis in the mouse. We found that Cenpj has two distinct roles in progenitors and in post-mitotic neurons. The loss of Cenpj function in cortical progenitors leads primarily to a defect in centrosome formation that results in abnormal spindle orientation during mitosis. In neurons, the loss of Cenpj function compromises radial migration and morphology. Moreover, we found that Cenpj expression in the embryonic cortex is induced by Ascl1, and that Cenpj is the main regulator of centrosome biogenesis and microtubule stability downstream of Ascl1 in the embryonic cerebral cortex.

\section{Results}

Cenpj is a transcriptional target of Ascl1. In a previous study of the genes regulated by the proneural protein Ascl1 in the telencephalon of embryonic day 14.5 (E14.5) mice, Ascl1 was found to be bound near the transcription start site of the Cenpj gene ${ }^{8}$. To determine whether this binding event (Fig. 1a) results in the regulation of Cenpj by Ascl1, we examined the expression of Cenpj in the telencephalon of E14.5 wild-type and Ascl1 null mutant embryos. Western blot (Fig. 1b,c) and immunocystochemistry analysis (Fig. 1d,e) showed that Cenpj protein is present at a reduced level in Ascl1 mutant than in wild-type cortex. Cenpj is expressed in proliferating cortical progenitors throughout interphase and mitosis and is downregulated in Ascl1 mutant cells throughout the cell cycle (Supplementary Fig. 1a). Quantitative PCR analysis showed a $50 \pm 5.7 \%$ reduction in Cenpj transcript in Ascl1 mutant telencephalon (Fig. 1f). Analysis by in situ hybridization showed that Cenpj transcripts are present in the ventricular zone (VZ), subventricular zone (SVZ) and the cortical plate $(\mathrm{CP})$ of the cerebral cortex in E14.5 wild-type embryos and are reduced in Ascl1 mutant embryos (Fig. 1g-i; Supplementary Fig. 1b). Together, these results suggest that Ascl1 regulates the Cenpj gene in the embryonic cortex and that it acts directly through interaction with a proximal regulatory element.

Cenpj is required for centrosome biogenesis. To determine the contribution of Cenpj to cortical development downstream of Ascl1, we used an acute loss-of-function approach by RNA interference. We selected a short-hairpin RNA (shRNA) that specifically knocked down Cenpj and reduced its expression to $\sim 50 \%$ (Supplementary Fig. 2a), and co-electroporated in utero the VZ cells of the cerebral cortex at E14.5 with this Cenpj shRNA and a plasmid expressing green fluorescent protein (GFP) to visualize electroporated cells. As progenitors in the telencephalon of Cenpj conditional null mutant mice (that is, with complete loss of function) have been shown to undergo apoptosis ${ }^{16}$, we first examined the presence of apoptotic cells among GFP ${ }^{+}$Cenpj knockdown cells (that is, with partial loss of function). There was no significant difference in numbers of activated caspase 3-positive cells between Cenpj shRNA- and control shRNAelectroporated brains, 1,2 and 3 days after electroporation (Supplementary Fig. 2b,c).

Cenpj is expressed in mitotic cells in the developing brain, suggesting that it may be required for normal proliferation of cortical progenitors. We therefore examined the divisions of electroporated cortical progenitors with an antibody against phosphohistone $\mathrm{H} 3$ ( $\mathrm{pH} 3$ ) to identify cells in the M-phase of the cell cycle. We observed that the fraction of eletroporated cells in mitosis was increased among Cenpj-silenced cells compared with control electroporated cells (Fig. $2 \mathrm{a}-\mathrm{c}$ ). We also analysed the cell cycle profile of Cenpj-depleted cells 2 days after electroporation by Hoechst staining and flow cytometry (Supplementary Fig. 2d). The results confirmed that Cenpj-depleted cells accumulate in the M-phase $(20.65 \pm 0.32 \%$ versus $16.73 \pm 1.1 \%$, mean \pm s.e.m. throughout the text, $n>200$ cells). Moreover, Cenpj-silenced progenitors had an aberrant distribution with an increased number of $\mathrm{pH}^{+}$cells that were scattered in the VZ (Fig. 2b,d), and many of these cells expressed the basal progenitor marker Tbr2 (Supplementary Fig. 2e-g). The presence of scattered $\mathrm{pH}^{+}$ cells in the VZ can be the result of a randomization of the cleavage plane of apical progenitors ${ }^{17-20}$. We therefore examined the cleavage plane of dividing apical progenitors when Cenpj was silenced, by double-labelling cells for $\mathrm{pH} 3$ and the centrosome marker $\gamma$-tubulin (Fig. 2e,f; Supplementary Fig. 2h). There was a strong increase in the fraction of Cenpj-silenced cells that divide with oblique cleavage planes and a reduction in cells dividing with a vertical plane, which were by far the most abundant in control electroporated cells (Fig. 2g,h). Randomization of the position of the mitotic spindle in Cenpj-silenced HeLa cells can result from a centrosome-formation defect ${ }^{13}$. We therefore examined centrosome formation in cortical $\mathrm{VZ}$ mitotic progenitors by measuring the centrosome area of $\gamma$-tubulin- or cdk5rap2-labelled centrosomes and observed a marked reduction in centrosome size in Cenpj-silenced cells $(43 \pm 3.5 \%, n>30$; Fig. 2i-k; Supplementary Fig. 2i,j), suggesting that Cenpj is required in apical progenitors for centrosome formation. Since Cenpj has also been shown to be required for centriole duplication in U2OS cells $^{13,14}$, we counted centrioles in centrin-GFP co-electroporated cells. The fraction of cells with a single centriole was greatly increased when Cenpj was silenced (Supplementary Fig. 2k,l). Labelling with alpha tubulin to detect microtubules revealed that Cenpj-depleted cortical progenitors maintained a normal bipolar organization of the mitotic spindle, but that the quantity of astral microtubules was decreased in comparison with control progenitors. In addition, Cenpj-depleted cells presented asymmetric spindles with lower alpha tubulin fluorescence associated with a smaller centrosome at one pole (Fig. 2l-n). 
a

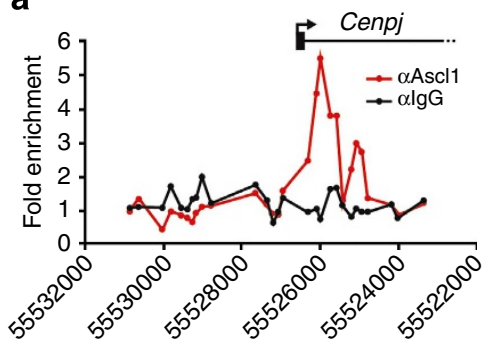

Chromosomal positions

d

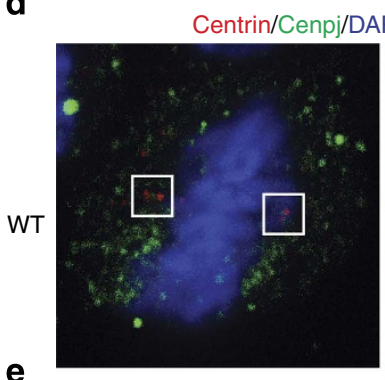

e

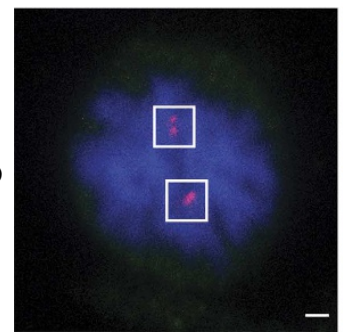

g
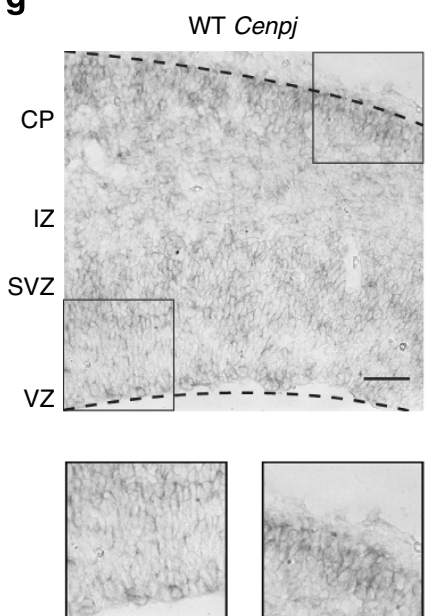

h

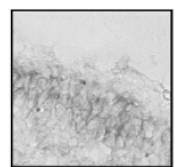

b

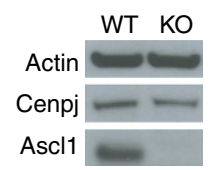

C

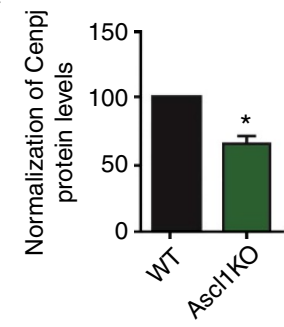

f
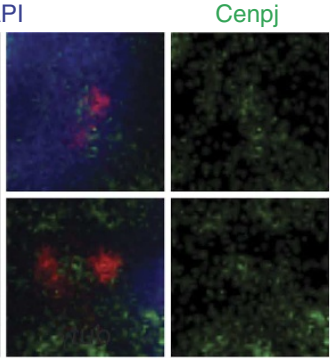

Centrin
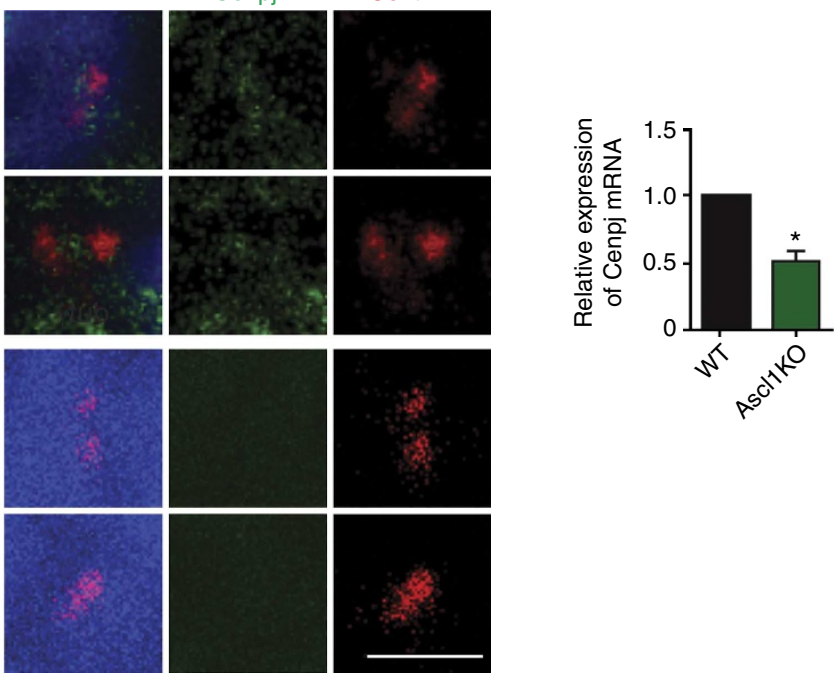
Ascl1 KO

i
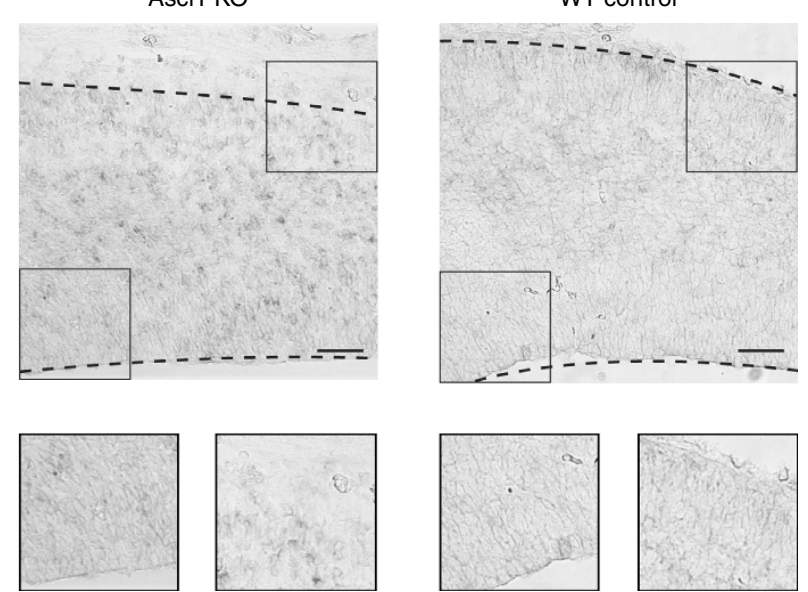

Figure 1 | Ascl1 directly regulates Cenpjexpression in the embryonic telencephalon. (a) Illustration of the Ascl1-binding signal in E14.5 telencephalic cells obtained by chromatin immunoprecipitation with promoter microarrays (chromatin immunoprecipitation (ChIP)-chip). The plot displays the ChIP enrichment ratio for Ascl1 (red) and control (black) samples in promoter regions. Black arrow indicates the transcription start site and direction of transcription. (b,c) Western blot analysis of Cenpj protein levels extracted from E14.5 Ascl1 mutants compared with wild-type (WT) telencephalon. Data presented as mean \pm s.e.m., $n=3$, Student's $t$-test, ${ }^{\star} P<0.05$. (d,e) Immunohistochemistry for Cenpj on coronal sections of WT and Ascl1 mutant E14.5 embryos. The Cenpj protein (d) is localized with the centrosome marker centrin in apical cortical progenitors and the signal is reduced in Ascl1 mutant progenitors (e). Scale bars, $1 \mu \mathrm{m}$. (f) Quantitative PCR analysis of Cenpj transcripts extracted from E14.5 Ascl1 mutants compared with WT telencephalon. Data presented as mean \pm s.e.m., $n=3$, Student's $t$-test, ${ }^{\star} P<0.05$. (g-i) In situ hybridization for Cenpj on coronal sections of the developing telencephalon at E14.5 from WT ( $\mathbf{g}, \mathbf{i})$ and Ascl1 knockout mouse (h) using an antisense Cenpj probe $(\mathbf{g}, \mathbf{h})$ and a control Cenpj sense probe (i). Expression of Cenpj was reduced in the ventricular zone and cortical plate of the Ascl1 mutant cortex. Scale bar, $100 \mu \mathrm{m}$. DAPI, 4',6-diamidino-2-phenylindole.

The most parsimonious interpretation of the multiple defects observed in the Cenpj-silenced progenitor is that Cenpj is primarily required in cortical progenitors for centrosome duplication, and when this process is disrupted, progenitors are delayed in their cell cycle progression and accumulate in the M-phase, the quantity of astral microtubules is reduced, the 
a

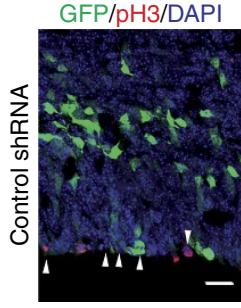

b
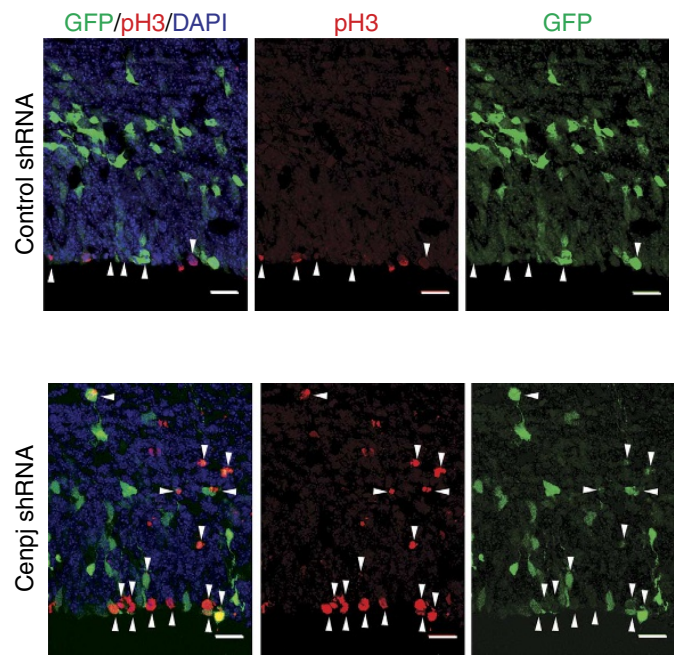

C

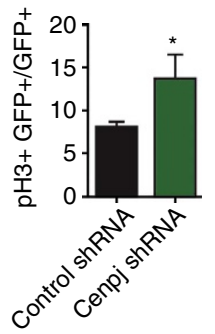

d

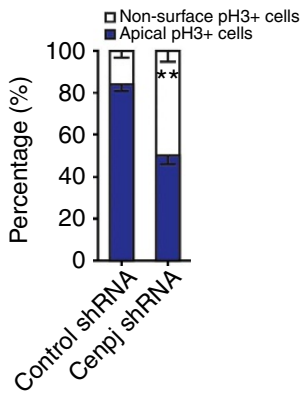

e

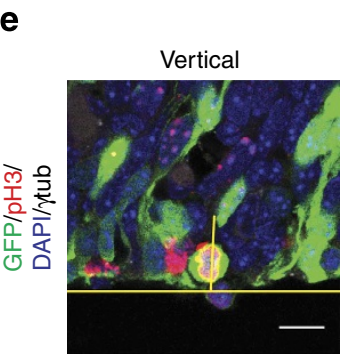

f

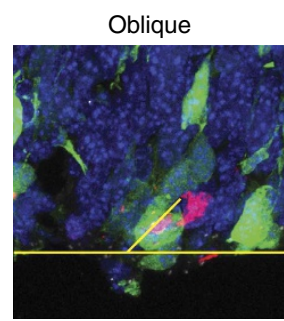

g
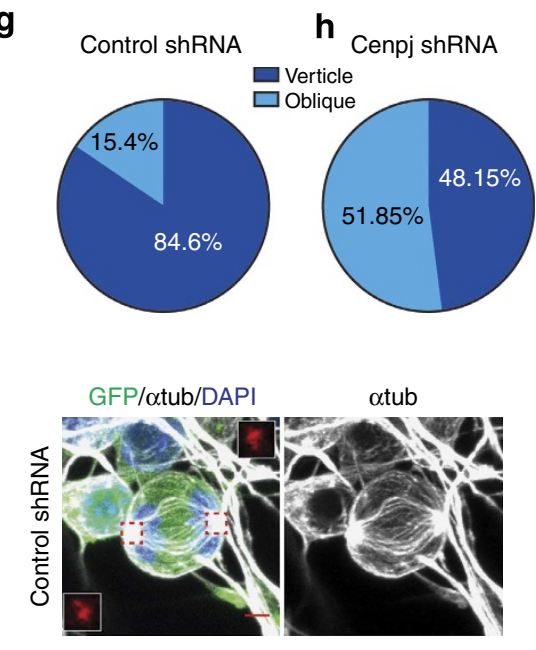

m

i $\mathrm{GFP} / \mathrm{pH} 3 /$

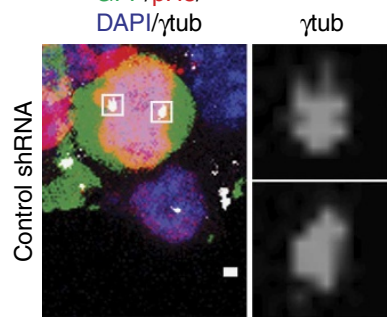

j

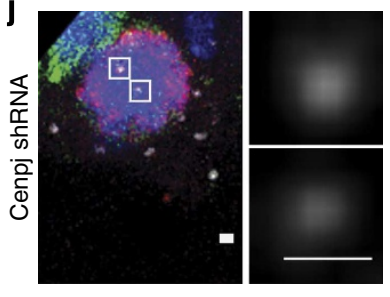

n

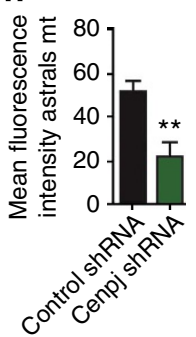

Figure 2 | Cenpj is required for the vertical divisions of cortical progenitors. (a,b) Immunostaining with a pH3 antibody to identify cells in the M-phase of the cell cycle of embryonic cortices 1 day after in utero co-electroporation at E14.5 of GFP and a control scrambled shRNA (a) or a Cenpj-specific shRNA (b). Arrowheads point to cells with co-localized pH3 and GFP signals. Scale bar, $20 \mu \mathrm{m}$. (c) Quantification of GFP + cells that also express pH3. Data presented here as the mean \pm s.e.m. from at least six sections prepared from three embryos obtained from two or three litters and $>200$ electroporated cells per embryo counted for each condition in this analysis and subsequent quantifications, unless stated otherwise. Student's $t$-test ${ }^{\star} P<0.05$. (d) Quantification of $\mathrm{pH}^{+} \mathrm{GFP}^{+}$cells located apically or scattered in the non-surface region 1 day after electroporation of the control or Cenpj shRNAs. Student's t-test ${ }^{\star \star} P<0.01$. (e,f) Examples of electroporated $\left(\mathrm{GFP}^{+}\right.$) mitotic apical progenitors in metaphase with vertical (e) or oblique (f) cleavage planes determined by co-labelling strong pH3 mitotic cells and $\gamma$-tubulin to mark the centrosomes and the apical surface. Scale bar, $10 \mu \mathrm{m}$. (g,h) Quantification of vertical (60-90 angles with a ventricular surface) and oblique (30-60 ) cleavage planes of mitotic apical progenitors 1 day after electroporation at E14.5 with control and Cenpj shRNAs. Control shRNA, $n=22$ cells; Cenpj shRNA, $n=20$ cells. Cells were analysed from at least six embryos obtained from three litters. (i,j) Electroporated (GFP ${ }^{+}$) mitotic $\left(\mathrm{pH}^{+}\right)$apical progenitors labelled for centrosome marker $\gamma$-tubulin. Insets show higher magnification of the centrosomes. The centrosomes of Cenpj shRNA-electroporated cells (j) have a reduced size compared with those of control cells (i). Scale bars, $1 \mu \mathrm{m}$. (k) Quantification of centrosome size in apical cortical mitotic cells 1 day after electroporation. Student's $t$-test ${ }^{\star \star \star} P<0.001$. (I,m) Electroporated (GFP ${ }^{+}$) progenitors labelled for centrosome marker Cdk5rap2 (red) and microtubule marker $\alpha$-tubulin (grey). Insets show higher magnification of the centrosomes. Cenpj shRNA-electroporated cells generate asymmetric spindle and less astral microtubules. Scale bar, $3 \mu \mathrm{m}$. (n) Quantification of astral microtubules' fluorescence intensity 2 days after electroporation. Student's $t$-test ${ }^{\star \star} P<0.01$. DAPI, 4',6-diamidino-2-phenylindole. 
orientation of the cleavage plane is randomized and daughter cells localize away from the ventricular surface. To determine whether the proliferation defects of Cenpj-depleted progenitors affect their rate of differentiation, we labelled electroporated cells for the early neuronal marker Tuj1, and found that Cenpj silencing resulted in fewer Tuj $1+$ neurons being generated 2 days after electroporation (Supplementary Fig. 2m-o).

Cenpj is required for cortical neuron migration. In addition to its expression and function in VZ progenitors, Cenpj is also expressed in post-mitotic neurons of the $\mathrm{CP}$ of the developing cerebral cortex (Fig. 1g; Supplementary Fig. 1b). Since a role of Cenpj in cortical neurons has not yet been studied, we investigated the consequence of Cenpj silencing in these cells by

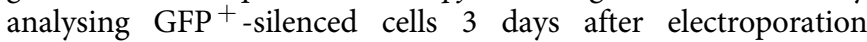
(Fig. 3a,b). Cenpj silencing resulted in a significant increase in the fraction of electroporated cells remaining in the intermediate zone (IZ) $\quad 39.3 \pm 3.4 \%$ Cenpj shRNA-electroporated versus $25.09 \pm 1.3 \%$ control shRNA-electroporated cells, $n>200$ cells), and a significant decrease in cells reaching the CP $(22.55 \pm 3.1 \%$ versus $40.7 \pm 3.4 \%, n>200$ cells; Fig. $3 \mathrm{a}, \mathrm{b}, \mathrm{e}$ ). Double labelling for GFP and the neuronal marker Tuj1 two days after electroporation showed that $64 \%$ of electroporated cells in the SVZ and $85 \%$ in the IZ were neurons (Supplementary Fig. 3d,e). Moreover, Cenpjsilenced neurons that had reached the $\mathrm{CP}$ displayed aberrant morphologies (Fig. 3g,h; Supplementary Fig. 3g-i). While control electroporated neurons in the $\mathrm{CP}$ displayed a characteristic bipolar morphology with a straight and thin leading process, Cenpj-silenced neurons presented an enlarged leading process and thin processes protruding from the cell body.

To determine whether the abnormal migration of Cenpjsilenced cells results indirectly from the defects observed in VZ progenitors, or reflects a distinct, cell autonomous function of Cenpj in neurons, we silenced Cenpj specifically in post-mitotic neurons. For this, we used a construct that expresses the Cenpj shRNA in a Cre-dependent manner (floxed stop-Cenpj shRNA) in the cortex of Nex-Cre mice, which express Cre only in post-mitotic cortical neurons ${ }^{21}$. Cells that had been silenced for Cenpj at the post-mitotic stage displayed migration defects ( $40.27 \pm 2.8 \%$ Cenpj shRNA-electroporated versus $23.80 \pm 1.05 \%$ control shRNA-electroporated cells in the IZ, $n>200$ cells; Fig. 3c,d,f) and morphological defects identical to those of cells silenced at the progenitor stage (Fig. 3i-k), demonstrating that Cenpj has a function in post-mitotic neurons and is directly required for neuronal migration in the cortex, in addition to its role in centrosome duplication in progenitors. To determine whether the neuronal migration defect of Cenpj-silenced neurons is transient or persists at later times, we examined at postnatal 2 the position neurons electroporated at E14.5. A significant fraction of Cenpj-depleted cells remained mislocated $(17.95 \pm 7.17 \%$ Cenpj shRNA- versus $48.70 \pm 3.1 \%$ control shRNA-electroporated cells in the upper CP (uCP), n>200 cells; Supplementary Fig. 3a-c), suggesting that Cenpj is required for the correct positioning of a large fraction of neurons in the $\mathrm{CP}$.

Cenpj promotes migration through microtubule destabilization. Migration of neurons in the $\mathrm{CP}$ is a cyclic process that includes a step of somal translocation during which the centrosome moves forward into the leading process and then pulls the nucleus to which it is connected by microtubules ${ }^{22}$. The increased thickness of the leading process of Cenpj-silenced neurons suggested that somal translocation might be impaired in these cells. To address this possibility, we co-electroporated a centrin-GFP construct together with Cenpj or control short interfering RNAs (siRNAs) and measured the distance between the centrosome and the tip of the nucleus (Fig. 3l,m) as an indication of centrosome-nucleus coupling. As expected, the centrosome was always found ahead of the nucleus in control experiments (Fig. 3l). In Cenpj-silenced neurons, however, not only the size but also the position of the centrosome were abnormal with some centrosomes located behind the front of the nucleus, suggesting that Cenpj is required for efficient coupling of the nucleus and centrosome in migrating neurons (Fig. 3m,n).

The translocation of the nucleus requires optimal microtubule dynamics. Both excessive stability and instability of the microtubules can alter the distance between the nucleus and centrosome and the thickness of the leading process, and both can interfere with neuronal migration ${ }^{23-27}$. The abnormal position of the centrosome and altered morphology of the leading process in Cenpj-silenced neurons could therefore result from a defect in microtubule stability. In U2OS and Hela cells, Cenpj overexpression has been reported to destabilize microtubules ${ }^{28,29}$. To investigate whether Cenpj knockdown also affects microtubule stability, we analysed the distribution of stable and unstable microtubules in Cenpj-silenced neurons isolated from the E14.5 cortex and maintained for 3 days in culture. The perinuclear tubulin cage could be labelled in control neurons with an antibody against tyrosinated tubulin, which marks highly dynamic microtubules ${ }^{30}$ (Fig. 4a). In Cenpj-silenced neurons, the tyrosinated tubulin labelling was weaker throughout the cells, including over the nucleus (Fig. 4b,c). In contrast, labelling with an antibody against acetylated tubulin, which marks stable microtubules, was enhanced in Cenpj-silenced neurons compared with control neurons, and stable microtubule bundles were found abnormally located over the nucleus, suggesting that the perinuclear tubulin cage is more rigid in Cenpj-silenced neurons than in control neurons (Fig. $4 \mathrm{~d}-\mathrm{f}$ ). To further examine microtubule stability in Cenpj-silenced neurons, we tested microtubule resistance to nocodazole-induced depolymerization $^{31,32}$ in Tbr1-expressing cortical neurons electroporated ex vivo and maintained in culture for 3 days (Supplementary Fig. 4a,b). As expected, treatment of control cortical neurons with nocodazole reduced microtubule stability, as visualized by acetylated tubulin labelling (Fig. $4 \mathrm{~g}$ ). In contrast, Cenpj-silenced neurons displayed greater resistance to the nocodazole treatment, as shown by the accumulation of acetylated tubulin in the processes and the presence of aberrant microtubule protrusions over the nucleus (Fig. 4h,i). Conversely, Cenpj overexpression resulted in a destablization of microtubules, as indicated by a sharp reduction of acetylated tubulin staining (Supplementary Fig. 4,d).

To further investigate the role of Cenpj in microtubule dynamics, we co-electroporated shRNAs with an end-binding protein 1 (EB1)-GFP construct to analyse EB1 comet live production, as described previously ${ }^{33}$ (Supplementary Movies 1 and 2). Cenpj silencing significantly reduced the number of comets (Supplementary Fig. 4,g) and the speed of tracks (Supplementary Fig. 4f,h) in cultured neurons 2 days after electroporation. Together, these results suggest that Cenpj is required to promote microtubule destabilization, which is essential for proper nuclear-centrosome coupling and nucleokinesis in migrating cortical neurons.

The PN2-3 protein domain is required for radial migration. The conserved PN2-3 domain of Cenpj has previously been shown to be both necessary and sufficient for the microtubule destabilization activity of the protein ${ }^{34}$. This domain has also been shown to be involved in centrosome formation ${ }^{13}$. To determine whether the PN2-3 domain contributes to Cenpj 
function in neuronal migration, we tested the capacity of a mutant version of Cenpj with a deleted PN2-3 domain (dPN2-3) to rescue the Cenpj knockdown phenotype when co-expressed with the Cenpj shRNA (Fig. 5d). As expected, co-electroporation of Cenpj shRNA with a full-length shRNA-resistant version of Cenpj fully rescued both the migration and morphological defects of Cenpj-silenced neurons (FL; Fig. 5a-c,f,h-j). In contrast, expression of shRNA-resistant Cenpj $d P N 2-3$ failed to rescue the defective migration $(23.25 \pm 1.2 \%$ versus $40.7 \pm 3.4 \%$ in the CP in control experiments, $n>200$ cells) and the abnormal leading process of Cenpj-silenced neurons, which were both as severe as for neurons electroporated with Cenpj shRNA alone a

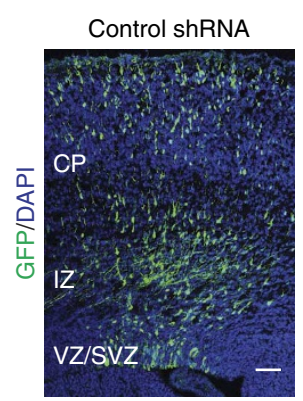

b

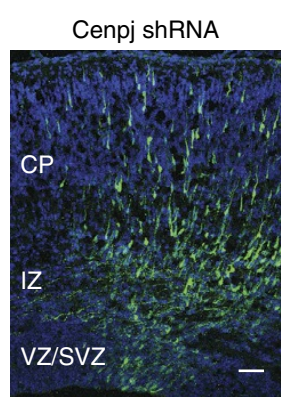

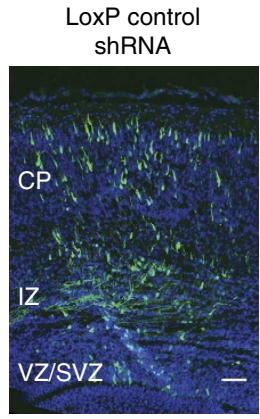

d

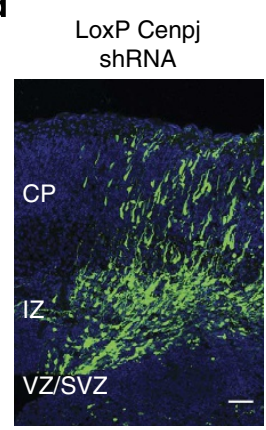

e

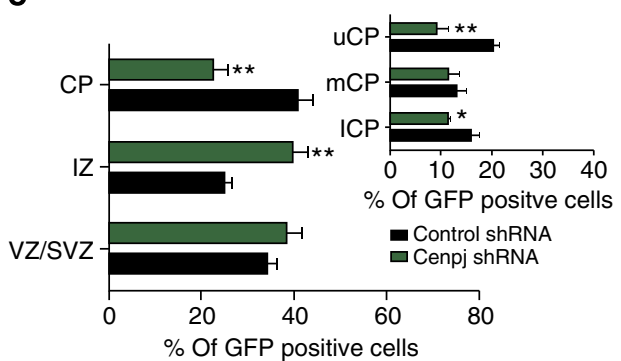

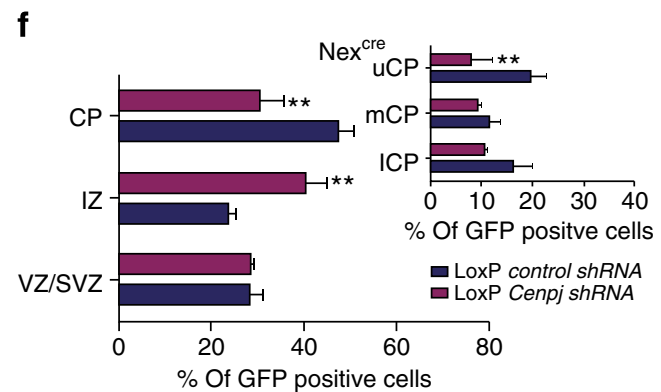

g

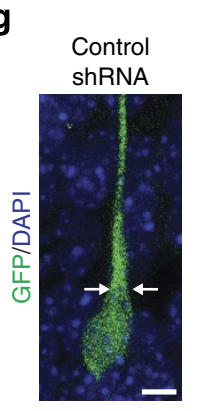

h

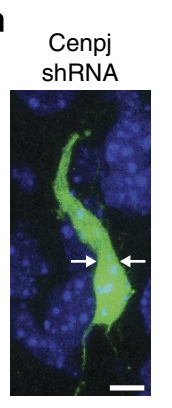

i

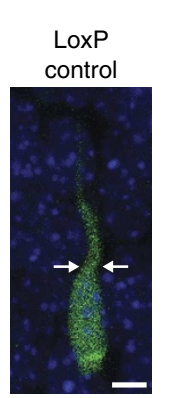

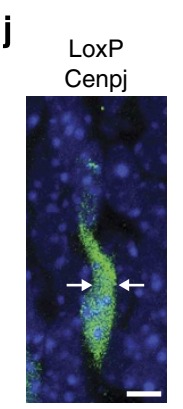

$\mathbf{k}$

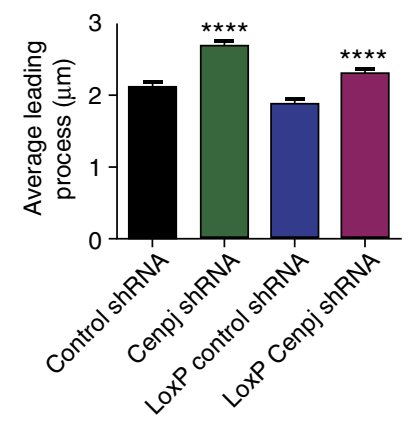

I

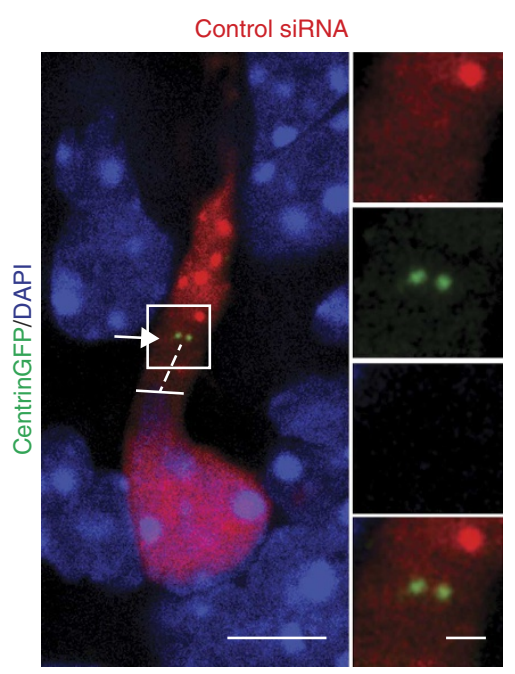

m

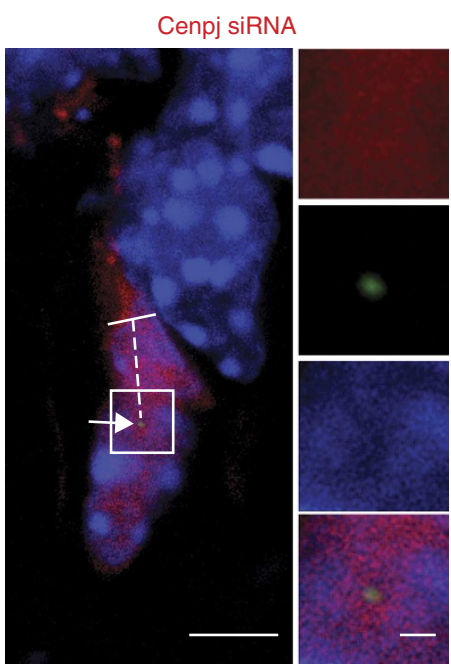

n

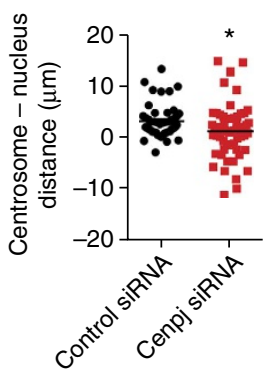


(Fig. 5d,g,l,m). The PN2-3 domain is therefore essential for Cenpj function in cortical neuron migration. Another domain of the Cenpj protein, $\mathrm{T}$ complex protein 10 (TCP), has been shown to be required for the centrosome duplication function of the protein ${ }^{13}$, but to be dispensable for its microtubule destabilization function ${ }^{13,29}$. To determine whether the TCP domain contributes to the activity of Cenpj in radial migration, we coelectroporated the Cenpj shRNA with a version of Cenpj that carries a deletion of this domain ( $d T C P)$. Cenpj $d T C P$ rescued the migratory behaviour and morphological defect of silenced neurons as efficiently as full-length Cenpj as shown in Fig. $5 \mathrm{~g}$ $(38.78 \pm 2.9 \%$ versus $40.7 \pm 3.4 \%$ in the $\mathrm{CP}$ in control experiments, $n>200$ cells) and Fig. $5 \mathrm{~m}$. We also examined whether the different Cenpj protein domains rescued the defects in microtubule stability and centrosome position observed in Cenpj-deficient neurons (Supplementary Fig. 5). Interestingly, only dTCP could rescue microtubule stability effects (Supplementary Fig. 5a-c) and centrosome-nucleus coupling (Supplementary Fig. 5d-f). However, neither TCP nor PN2-3 rescued the centrosome size or centriole number defects of Cenpjdeficient progenitors (Supplementary Fig. 2i-2l). Together, these results suggest that Cenpj promotes nuclear translocation and neuronal migration in the developing cortex by destabilizing microtubules through the activity of its PN2-3 domain, independently of its role in centrosome formation.

Cenpj is an effector of Ascl1. The foregoing data demonstrate that Cenpj has important roles in both progenitor cells and neurons during cortical development. Since Cenpj expression in the embryonic cortex is regulated by the proneural protein Ascll (Fig. 1), Cenpj might contribute significantly to Ascll functions in cortical development. To address this possibility, we compared the defects caused by Ascll loss of function in cortical progenitors with those displayed by Cenpj-silenced progenitors. Labelling for $\gamma$-tubulin and measuring the centrosome area showed that centrosomes in mitotic cortical cells were smaller in Ascl1 mutant progenitors than in wild-type progenitors $(60.36 \pm 6 \%, P<0.001$, Student's $t$-test, $n>30$ per condition; Fig. $6 \mathrm{a}-\mathrm{c})$. To determine whether Cenpj is the main effector of Ascl1 in the regulation of centrosome formation, we overexpressed Cenpj in Ascl1-silenced apical progenitors and we found that this fully corrected the size of the centrosome (Fig. $6 \mathrm{~d}-\mathrm{f}$ ).

The cleavage plane of cortical apical progenitors has previously been shown to be essentially random in Ascll mutant E14.5 embryos instead of mostly vertical in wild-type embryos, similar to the defects observed in Cenpj-silenced progenitors (Figs $2 \mathrm{f}$ and $6 \mathrm{~g})^{5}$. To determine whether Cenpj contributes to the function of Ascll in regulating spindle orientation, we overexpressed Cenpj in Ascl1-silenced apical progenitors. We found that this fully corrected the abnormal orientation of the progenitor divisions to mostly vertical angles (Fig. 6h).

Ascl1 silencing in cortical progenitors results 3 days later in accumulation of their progeny in the IZ and in their depletion from the $\mathrm{uCP}^{6}$ (Fig. 7a,b,d), a defect similar to that observed with Cenpj-silenced neurons. Also similar to Cenpj-silenced cells, Ascl1-silenced neurons in the CP exhibit an enlargement of the leading process (Fig. 7e,f) and microtubules also appear bundled, as shown by the accumulation of acetylated tubulin over the nucleus after nocodazole treatment (Fig. 7i,j). Co-electroporation of Cenpj with the Ascl1 shRNA corrected the abnormal accumulation of Ascl1-silenced cells in the IZ $(37.0 \pm 2.8 \%$ Ascl1 shRNA versus $25.7 \pm 1.13 \%$ Ascl1 shRNA + pCMVCenpj-electroporated versus $25.09 \pm 1.37 \%$ control shRNAelectroporated cells, $n>200$ cells; Fig. $7 c$,d). The abnormal morphology of the leading process of Ascl1-silenced cells (Fig. $7 \mathrm{~g}, \mathrm{~h}$ ) and the accumulation of acetylated tubulin staining over the nucleus were also corrected by overexpression of Cenpj (Fig. 7k,l). However, the migration of Ascll-silenced neurons to the upper part of the CP was not corrected by overexpression of Cenpj (Fig. 7d), suggesting that another gene regulated by Ascl1 is required for this later phase of migration.

Altogether, these results demonstrate that Cenpj regulates several crucial steps of neurogenesis in the cerebral cortex downstream of Ascl1, including centrosome biogenesis, the orientation of progenitor cleavage planes and the destabilization of microtubules and nuclear translocation during neuronal migration.

\section{Discussion}

The transcription factor Ascl1 is expressed in progenitors of the embryonic cerebral cortex ${ }^{4}$ and has specific functions in cortical neurogenesis, although it is not directly involved in the neuronal commitment and differentiation of progenitors in this brain region, which is a role taken by another proneural protein, Neurog2 (refs 35,36). Ascl1 is required for the proper orientation of the progenitor divisions in the $\mathrm{VZ}^{5}$ and for the migration of cortical neurons through the $\mathrm{CP}^{6}$. The pathways that mediate these functions of Ascll have begun to be characterized. Here we identify the microcephaly protein Cenpj as a target of Ascll involved in multiple steps of cortical neurogenesis. Below, we discuss first the function of Cenpj in dividing cortical progenitors and then its role in migrating cortical neurons.

Our demonstration that Cenpj is required in cortical progenitors for centrosomes to reach a normal size is in agreement with previous studies examining the effect of Cenpj silencing in HeLa, U2OS and mouse embryonic fibroblast cells (MEFs). These earlier studies also showed that the centrosome biogenesis defect leads to

Figure 3 | Silencing Cenpj alters neuronal migration and morphology. (a-d) Analysis of radial migration by GFP labelling of cortices 3 days after in utero co-electroporation at E14.5 with GFP and control shRNA (a), Cenpj shRNA (b). pCALSL control shRNA (c) and conditionally activable pCALSL_Cenpj shRNA (d). pCALSL control and_Cenpj shRNA were electroporated in Nex-cre embryos. Scale bar, $50 \mu \mathrm{m}$. (e,f) Quantification of the migration defect of neurons silenced for Cenpj in VZ progenitors (e) or in Nex + neurons (f) by measuring the percentage of GFP ${ }^{+}$cells that have reached the different zones of the cortex 3 days after electroporation. Cells silenced for Cenpj at both progenitor and neuronal stages accumulate more in the IZ and less in the upper CP than control cells. Student's t-test ${ }^{\star} P<0.05,{ }^{\star \star} P<0.01$. ( $\mathbf{g}$-j) Cenpj-depleted neurons silenced at both progenitor $(\mathbf{h})$ and neuronal stages $(\mathbf{j})$ display abnormal enlargement of the leading process 3 days after electroporation compared with control neurons $(\mathbf{g}, \mathbf{i})$. Scale bar, $5 \mu \mathrm{m}$. (k) Quantification of the thickness of the leading process performed 3 days after electroporation, as described in the Methods section. Cenpj-depleted neurons have a thicker leading process. Three embryos analysed for each condition; control shRNA, $n=177$ cells; Cenpj shRNA, $n=200$ cells. Student's $t$-test ${ }^{\star \star \star \star} P<0.0001$. (I,m) Analysis of the distance between the centrosome and the nucleus in migrating cortical neurons 3 days after in utero co-electroporation at E14.5 of control or Cenpj shRNAs together with pCIG2-Centrin2-Venus for labelling of the centrioles and pCMV-RFP construct to mark electroporated cells. The nuclei are labelled with 4',6-diamidino-2-phenylindole (DAPI). Insets show higher magnification of the boxed areas with separate colour channels. Arrows point to the centrosome, dashed lines mark the distance between the centrosome and the tip of the nucleus, marked by a full line. Scale bar, $3 \mu \mathrm{m}$ (main panels) and $1 \mu \mathrm{m}$ (insets). (n) Quantification of the distance between the centrosome and the nucleus in migrating cortical neurons. Negative values correspond to centrosomes located below the tip of the nucleus. Cenpj-depleted neurons present more negative centrosome-nucleus distances than control neurons. Three embryos analysed for each condition; control shRNA, $n=47$ cells; Cenpj shRNA, $n=60$. Student's $t$-test ${ }^{\star} P<0.05$. 
a

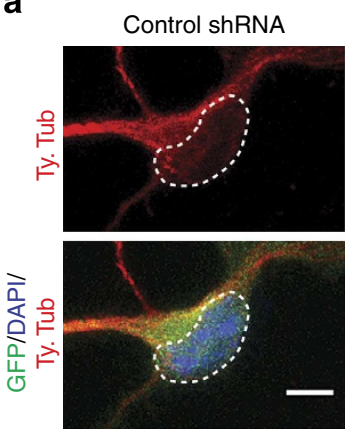

d

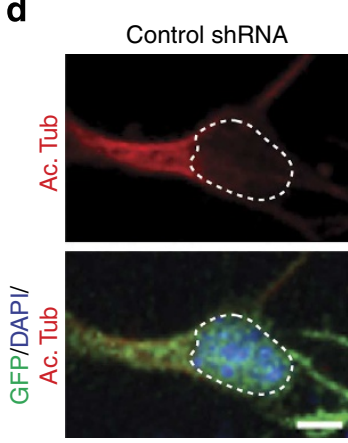

g

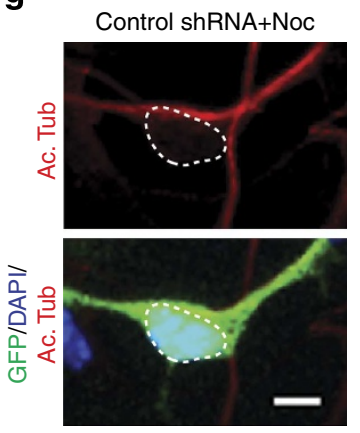

b

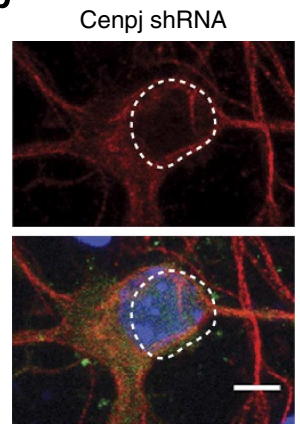

e

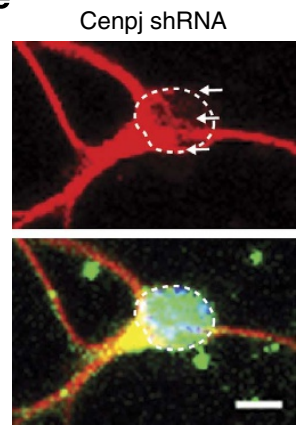

h

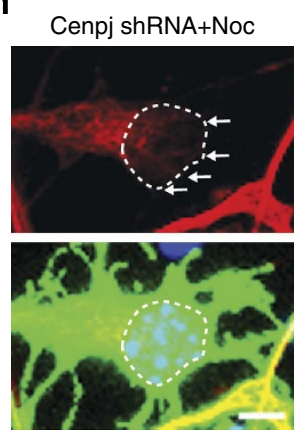

C

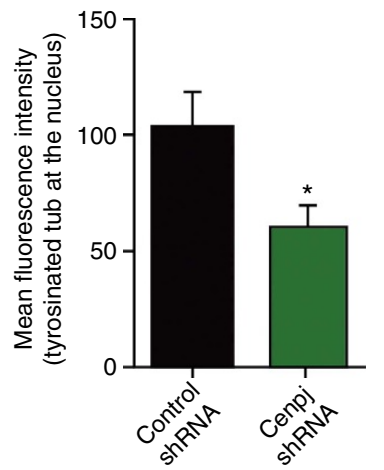

f

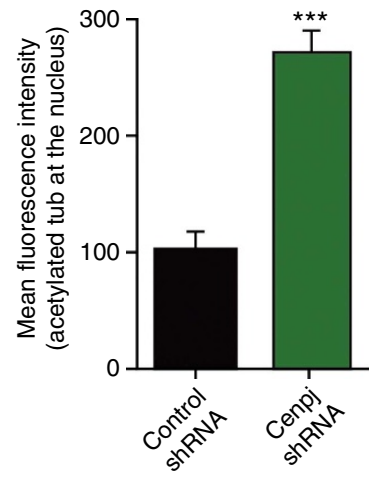

i

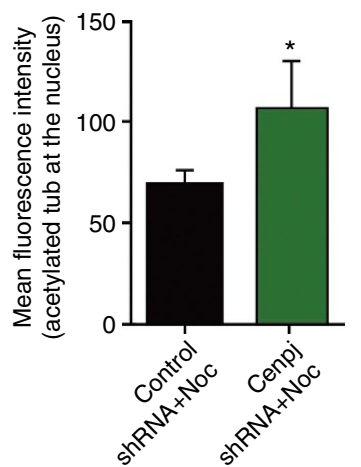

Figure 4 | Cenpj silencing disrupts microtubule dynamics. (a,b,d,e) Analysis of dynamic tyrosinated microtubules (a,b) and stable acetylated microtubules (d,e) in neurons cultured in vitro for 3 days after ex vivo electroporation of GFP and control (a,d) or Cenpj shRNAs (b,e). White line shows 4',6-diamidino-2-phenylindole (DAPI) limits. Scale bar, $3 \mu \mathrm{m}$. (c,f) Quantification of the mean fluorescence intensity of tyrosinated tubulin (c) and acetylated tubulin (f) overlapping the nucleus labelled with DAPI. Cenpj silencing results in a reduction of dynamic microtubules and an increase in stable microtubules in the microtubule cage enveloping the nucleus. The analysis was performed in three independent cultures; control shRNA, $n=44$ cells; Cenpj shRNA, $n=70$ (e), control shRNA, $n=60$ cells; Cenpj shRNA, $n=55$ (f). Student's $t$-test ${ }^{\star} P<0.05 ;{ }^{\star \star \star} P<0.001$. (g,h) Effect of nocodazole on stable acetylated microtubules in neurons cultured in vitro for 3 days after ex vivo electroporation of GFP and control or Cenpj shRNAs (g,h). White line shows DAPI limits. Scale bar, $3 \mu \mathrm{m}$. (i) Quantification of acetylated tubulin labelling co-localized with the nucleus in nocodazole-treated cultures. Cenpj silencing results in an increase of stable microtubules in the microtubule cage compared with shRNA control-treated cells (arrows). Analysis of three independent cultures; control shRNA, $n=40$ cells; Cenpj shRNA, $n=42$. Student's $t$-test ${ }^{\star} P<0.05$.

mitotic spindle abnormalities ${ }^{13,14,16}$, which we also observed in Cenpj-depleted cortical progenitors in this study. Similarly, the analysis of mice mutant for the centrosome-associated proteins Treacle and Nde1 and the cell cortex-associated protein Lgn have shown that randomization of the plane of progenitor divisions results in mitotic progenitors being located away from the apical cortical surface $17,18,20$. Abnormal angles of progenitor divisions are likely to be also at the origin of the displaced dividing Cenpjsilenced progenitors. A large fraction of these scattered progenitors acquire a basal progenitor fate, as shown by Tbr2 expression and as reported previously ${ }^{37}$. It seems less likely that Cenpj silencing results in an increased proliferation of basal progenitors because this would result in an increase in neuronal production, and we observe instead a decrease.

Cenpj hypomorphic mice present a reduction in brain size, but not an increase in apoptosis in the dorsal telencephalon ${ }^{15}$. Similarly, we did not observe an increase in cell death following partial loss of function of Cenpj in the developing cerebral cortex 
a

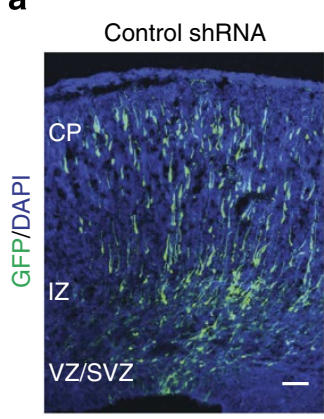

b

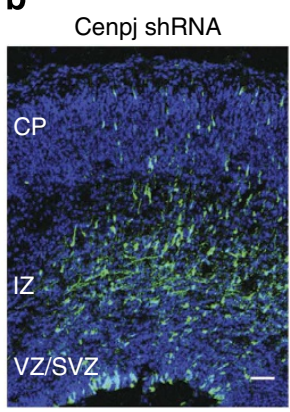

C

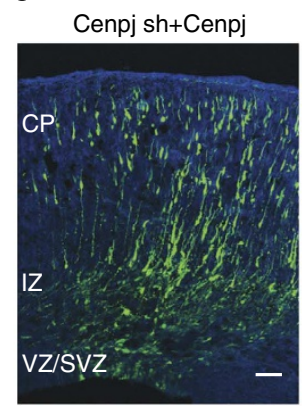

d

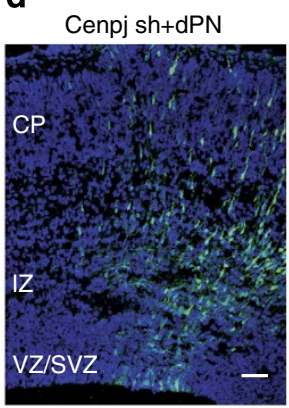

e

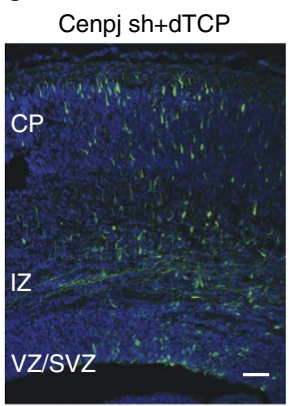

f

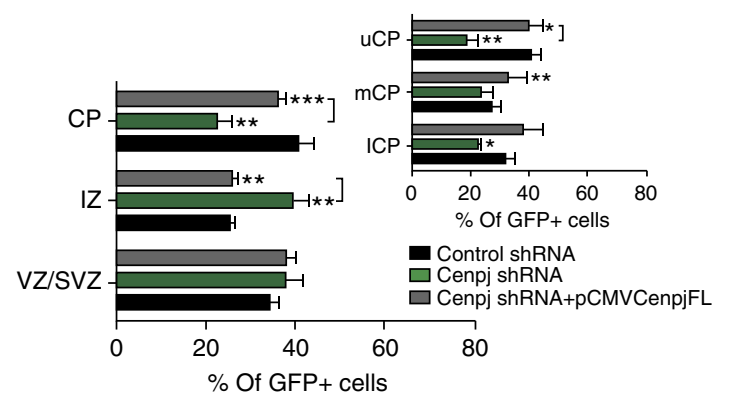

g $h$


$\bar{a}$
0
0
0
0
0

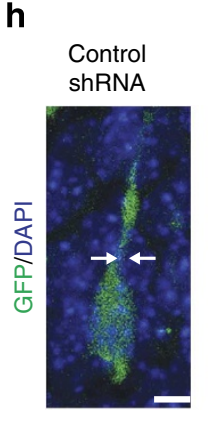

\section{i} Cenpj shRNA

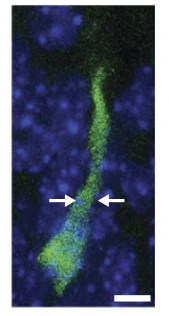

j Cenpj sh+ Cenpj

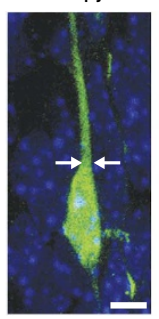

$\mathbf{k}$ Cenpj sh+ dPN

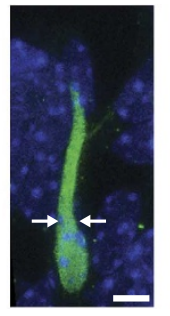

I

Cenpj sh+ dTCP

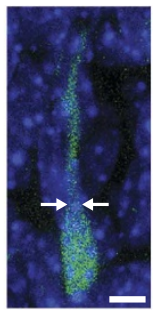

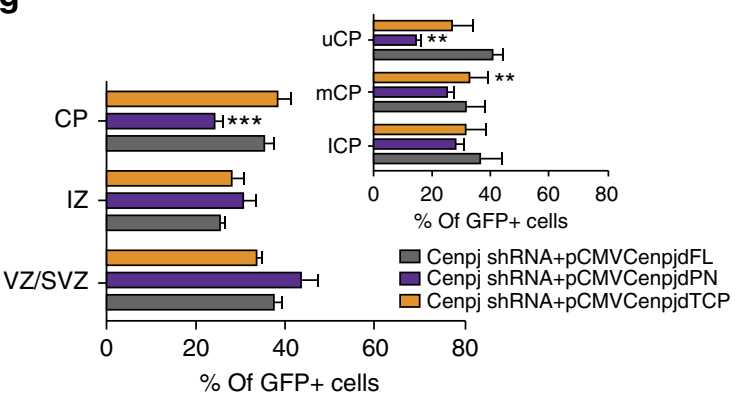

m

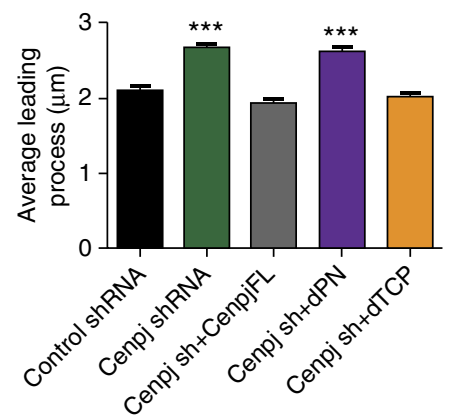

Figure 5 | Microtubule-destabilizing domain of Cenpj is required for migration. (a-e) Analysis of radial migration in cortices 3 days after co-electroporation of GFP with a control shRNA (a), Cenpj shRNA alone (b), Cenpj shRNA together with a full-length Cenpj expression construct (pCMV-Cenpj, c), Cenpj shRNA with a truncated Cenpj construct lacking the microtubule-destabilizing domain PN2-3 (pCMV-Cenpj dPN2-3, d) or Cenpj shRNA with a truncated Cenpj construct lacking the domain TCP (pCMV-Cenpj dTCP, e). Scale bar, $50 \mu \mathrm{m}$. (f,g) Quantification of the migration defects of $\mathrm{GFP}^{+}$cells in the different zones of the cortex. The migration defect of Cenpj-depleted cells is rescued by overexpression of full-length Cenpj (f) and Cenpj dTCP but not of Cenpj dPN2-3 (g). Student's $t$-test ${ }^{\star} P<0.05$; ${ }^{\star \star} P<0.01$; ${ }^{\star \star \star} P<0.001$. (h-I) Analysis of leading process thickness in neurons in the CP co-electroporated with GFP and Control shRNA (h), with Cenpj shRNA (i) and Cenpj shRNA and Cenpj full length (j) or Cenpj shRNA and Cenpj dPN (K) or Cenpj shRNA and Cenpj dTCP (I). Scale bar, $5 \mu \mathrm{m}$. (m) Quantification of leading process thickness in GFP + neurons. The leading process enlargement observed in Cenpj-depleted neurons is rescued by co-expression of Cenpj shRNA with Cenpj full length and Cenpj dTCP but not by Cenpj dPN. Three embryos analysed for each condition; control shRNA, $n=177$ cells; Cenpj shRNA, $n=200$ cells; Cenpjsh + Cenpj FL, $n=157$ cells; Cenpj shRNA + Cenpj dPN2-3, $n=274$ cells; Cenpj shRNA + Cenpj dTCP, $n=211$ cells. Student's $t$-test ${ }^{\star \star \star} P<0.001$. DAPI, 4',6-diamidino-2-phenylindole.

by shRNA-mediated knockdown. However, embryos with a complete elimination of Cenpj present a widespread, p53dependent cell death phenotype ${ }^{16}$. We therefore suggest that the severity of the phenotype resulting from Cenpj deficiency is dependent on the level of Cenpj protein expressed.

Altogether, based on our data and on previous studies in different systems, we propose that the primary role of Cenpj in progenitors is in the elongation of the procentrioles during centrosome duplication, as well as in the recruitment of the pericentriolar material as a scaffolding protein ${ }^{13,14,38,39}$. As a consequence of Cenpj depletion, the centrioles are shorter and the pericentriolar material is not recruited properly, resulting in a smaller and fragmented centrosome. This primary centrosome biogenesis defect leads to abnormal centrosome duplication, spindle malformation and mitotic cleavage randomization.
While the role of Cenpj in regulating centrosome formation and mitotic spindle orientation had been previously reported in cultured cells, its involvement in cell migration that we report in cortical projection neurons is novel. The role of Cenpj in the radial migration of cortical neurons involves the destabilization of the microtubule cage that surrounds the nucleus, thus promoting efficient centrosome-nucleus coupling. This is clearly independent from the role of Cenpj regulation of centrosome size in progenitors, because the migratory defect is observed specifically when Cenpj is silenced in neurons. Moreover, it is rescued by a version of Cenpj that lacks the TCP domain, involved in centrosome formation, while it is not rescued by a version lacking the microtubule-destabilizing domain PN2-3 (ref. 13). The PN2-3 domain inhibits microtubule assembly in vitro by binding and sequestering single $\alpha / \beta$-tubulin dimers ${ }^{28,40}$. A recent 
a
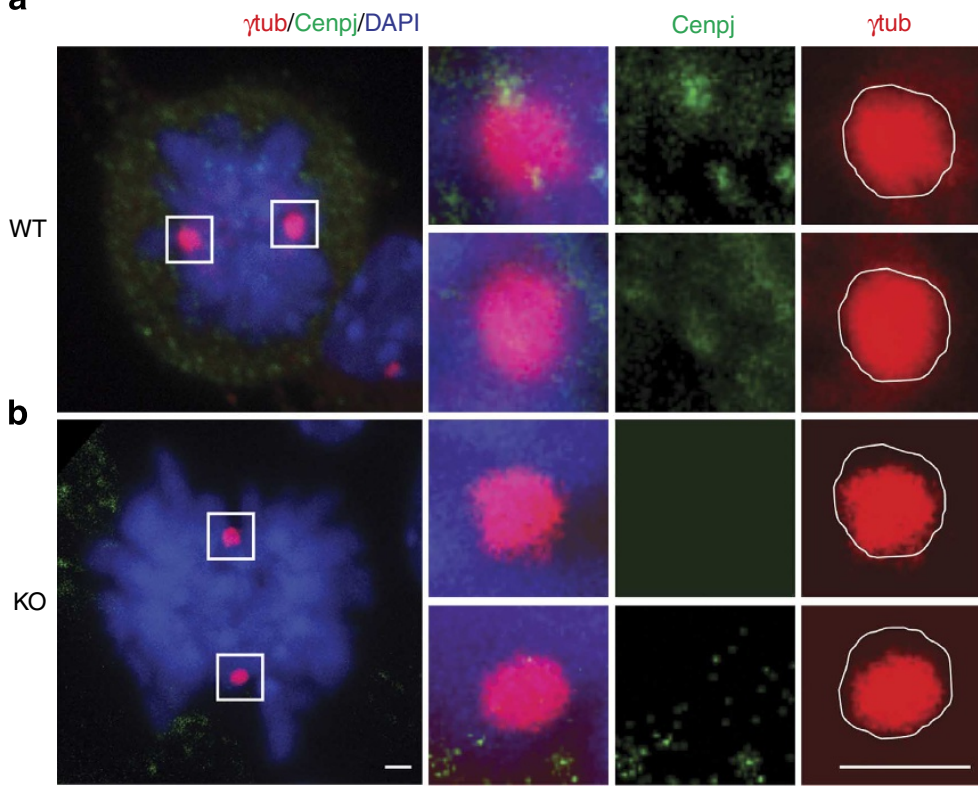

c

d

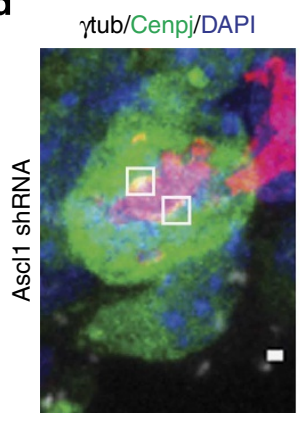

g

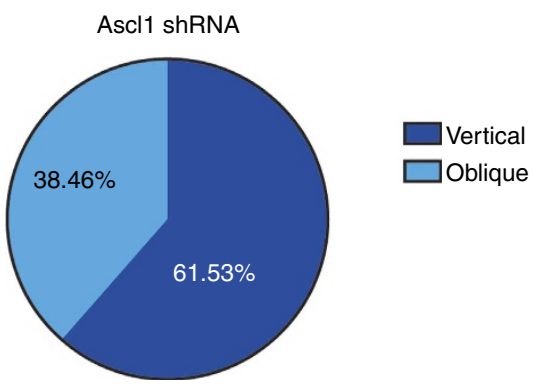

e

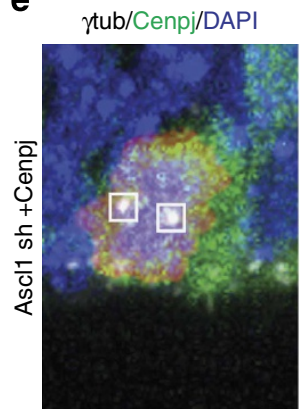

$\gamma$ tub

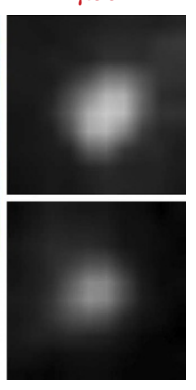

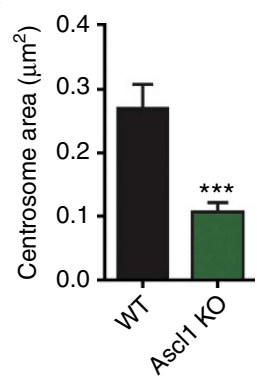

f

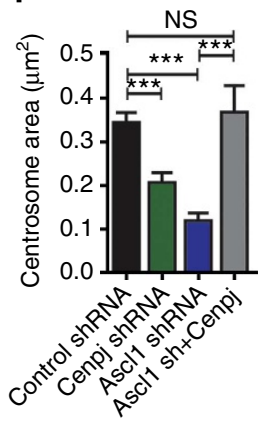

h

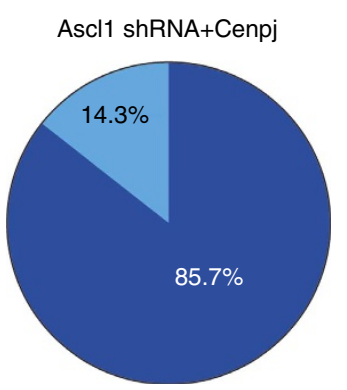

Figure 6 | Cenpj is the main effector of Ascl1for centrosome biogenesis. (a,b) Labelling of centrosomes in apical cortical progenitors from wild-type (WT) (a) or Ascl1 null embryos (b) with an antibody to $\gamma$-tubulin. Insets to the right show higher magnification of the boxed areas with separate colour channels. Scale bars, $1 \mu \mathrm{m}$. (c) Quantification of the area of the centrosomes in WT and Ascl1 null embryos labelled with an antibody to $\gamma$-tubulin. Three embryos analysed for each condition. Student's $t$-test ${ }^{* \star *} P<0.001$. (d,e) Analysis of the centrosome area of GFP + -dividing apical progenitors electroporated 1 day earlier in utero with Ascl1 shRNA (d) or an Ascl1 shRNA plus a pCMV-Cenpj construct (e) labelled for GFP (green), pH3 (red) and $\gamma$-tubulin (grey). Insets to the right show higher magnification of the boxed areas with $\gamma$-tubulin (grey) channels. Scale bars, $1 \mu \mathrm{m}$. (f) Quantification of the area of the centrosomes in control shRNA-electroporated progenitors, Cenpj shRNA, Ascll shRNA and Ascl1 shRNA + pCMV-Cenpj-electroporated progenitors, labelled with $\gamma$-tubulin. The centrosomes are smaller in Ascl7-silenced progenitors and this defect is rescued in Ascl1-silenced cells co-electroporated with a Cenpj expression vector. Student's t-test ${ }^{\star \star \star} P<0.001$. (g,h) Percentage of cells electroporated with Ascl1 shRNA (g) and Ascl1 shRNA + pCMV-Cenpj (h) having a vertical $\left(60-90^{\circ}\right)$ or oblique $\left(30-60^{\circ}\right)$ cleavage angle. Ascl1 shRNA, $n=33$ cells; Ascl1 shRNA + Cenpj, $n=24$ cells. Cells were analysed from at least six embryos obtained from three litters. DAPI, 4',6-diamidino-2-phenylindole; NS, not significant.

study also reported cortical neuron migration defects in Cenpj null mutant embryos, in which progenitor cell death had been rescued by $\mathrm{p} 53$ deletion, manifested by the formation of small cortical heterotopias ${ }^{41}$. Cenpj thus joins the list of microtubuledestabilizing proteins that have been shown to be required for maintenance of a dynamic microtubule cage, the regulation of the thickness of a leading process and proper positioning of the centrosome with respect to the nucleus, including the microtubule-severing proteins katanins and the microtubule disassembly proteins stathmins ${ }^{26,42-44}$. Other microcephaly proteins, such as ASPM and Ndel1, also have essential roles in progenitor divisions and post-mitotic neurons ${ }^{45-48}$, and have 
been shown to act by interacting with motor proteins. Ndle1 interacts with Lis1 and the dynein complex ${ }^{49}$, while the Caenorhabditis elegans ASPM orthologue ASPM-1 interacts with dynein ${ }^{50}$ and the Drosophila ASPM orthologue Asp interacts with myosin $\mathrm{II}^{51}$. Whether Cenpj also influences motor protein activity remains to be investigated.

Analysis of the Drosophila mutant for the Cenpj homologue Sas-4 did not reveal any brain-organization defects ${ }^{52}$. We show here that a deficit in Cenpj results in abnormal positioning of a significant fraction of cortical neurons at postnatal stages. Whether loss of Cenpj also results in cortical connectivity defects and abnormal brain function is an important question that remains to be addressed.

The regulation of Cenpj expression in the cerebral cortex by Ascl1 and the similarities of the migratory defects of Cenpj- and Ascl1-deficient neurons suggested that Cenpj might mediate some aspects of Ascl1 function in neuronal migration. Indeed, coexpression of Cenpj with an Ascl1 shRNA rescued the abnormal accumulation of Ascl1-silenced neurons in the IZ, thus establishing the importance of Cenpj activity in the pro-migratory programme driven by Ascll. However, the rescue of Ascl1silenced neurons was not complete since cells did not migrate to a

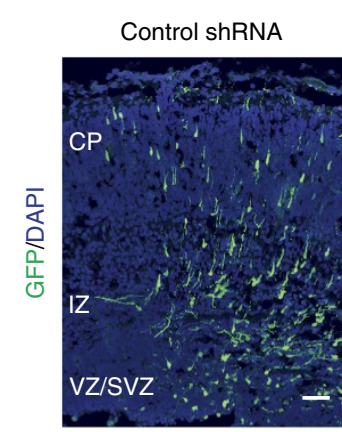

b

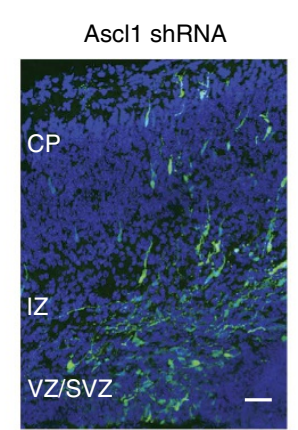

C Ascl1 shRNA + pCMV Cenpj

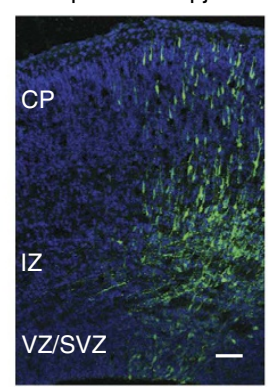

e

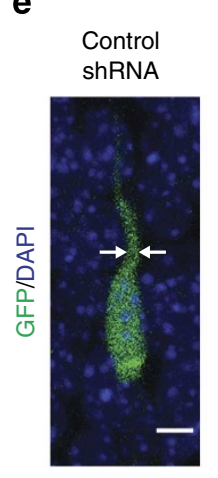

f

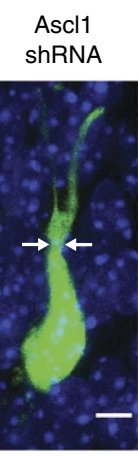

g

Ascl1 sh +Cenpj

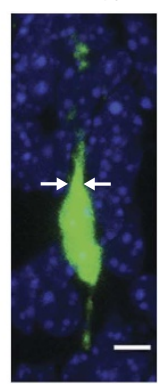

d

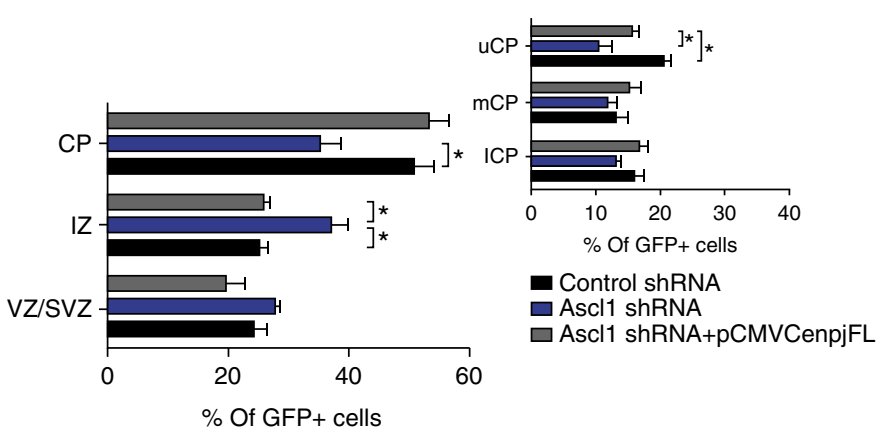

h

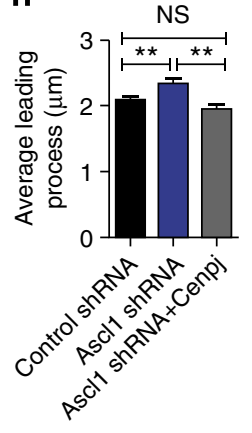

i

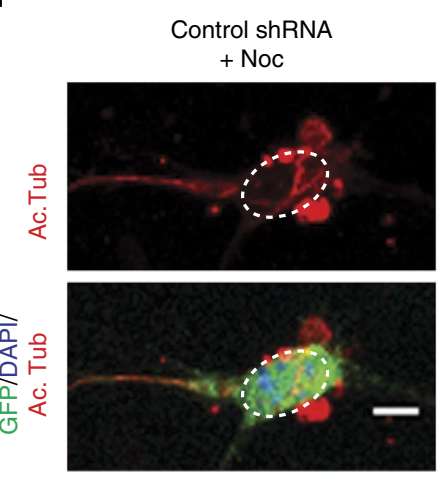

j

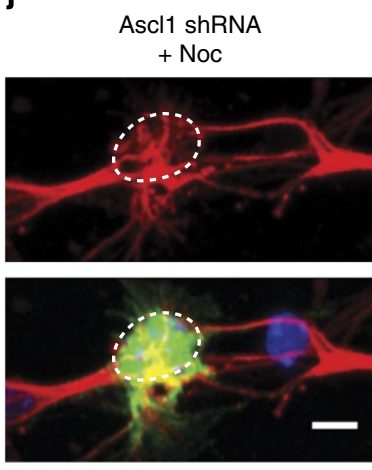

k

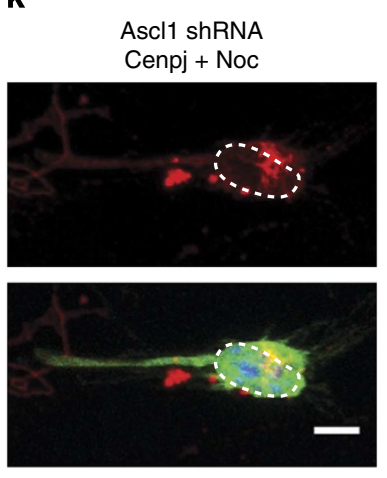

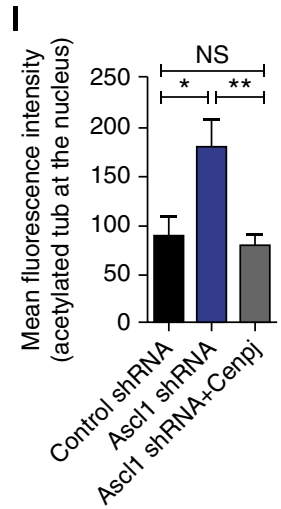

Figure 7 | Cenpj is the main effector of Ascl7for microtubule regulation. (a-c) Analysis of radial migration 3 days after electroporation of E14.5 cortices with GFP and a control shRNA, Ascl1 shRNA or Ascl1 shRNA + pCMV-Cenpj. Scale bar, $50 \mu \mathrm{m}$. (d) Quantification of the migration of GFP + cells in the different zones of the cortex. The accumulation of Ascl7-silenced cells in the IZ but not their depletion from the uCP was rescued by overexpression of Cenpj. Student's $t$-test ${ }^{\star} P<0.05(\mathbf{e}-\mathbf{g})$. Abnormal leading-process enlargement of Ascl7-silenced neurons in the CP. Scale bar, $5 \mu \mathrm{m}$. (h) Quantification of leading process thickness. Three embryos analysed for each condition; control shRNA, $n=177$ cells; Ascl1 shRNA, $n=128$ cells; Ascl1 shRNA + pCMV-Cenpj, $n=239$ cells. Student's $t$-test ${ }^{\star \star} P<0.01$. (i-k) Nocodazole treatment of cultured neurons 3 days after electroporation. Ascl1 silencing preserved a higher level of acetylated tubulin labelling in the cell body and leading process than control shRNA, and overexpression of Cenpj restored the acetylated tubulin-labelling intensity. Scale bar, $5 \mu \mathrm{m}$. (I) Quantification of acetylated tubulin labelling co-localized with the nucleus. Three independent cultures analysed for each condition; control shRNA, $n=40$ cells; Ascl1 shRNA, $n=53$; Ascl1 shRNA + pCMV-Cenpj, $n=37$. Student's $t$-test ${ }^{\star} P<0.05 ;{ }^{\star \star} P<0.01$. DAPI, 4',6-diamidino-2-phenylindole; NS, not significant. 
the uCP as efficiently as control cells. This result was expected since Ascl1 also regulates the cortical expression of Rnd3, a constitutively active small guanosine triphosphate-binding protein that promotes cortical neuron locomotion in the CP by inhibiting RhoA activity and destabilizing the actin cytoskeleton ${ }^{6}$. Together, our studies show that Ascl1 controls neuronal migration by regulating both the actin and microtubule cytoskeletons, via Rnd3 and Cenpj, respectively.

The function of Cenpj at two crucial steps of cortical development likely explains the long-term consequences of Cenpj deficiency in the formation and function of the cerebral cortex. Our work provides insights into the mechanisms of Cenpj function and regulation. It implicates the proneural factor Ascl1 and the microcephaly protein Cenpj in neuronal migration, thus improving our understanding of human microcephalies and microlissencephalies.

\section{Methods}

Animals. Mice were housed, bred and treated according to the guidelines approved by the Home Office under the Animal (Scientific procedures) Act 1986. The following mouse lines were used and genotyped, as described previously: Ascl1 ${ }^{\text {delta53}}$; Nex ${ }^{\text {Cre21. }}$.

Cell culture. Mouse embryonic teratocarcinoma P19 were cultured in high-glucose DMEM (Invitrogen) supplemented with $10 \%$ fetal bovine serum, $2 \mathrm{mM}$ glutamine and $1 \%$ penicillin/streptomycin. NS5 mouse cells and cortical progenitors from E14.5 wild-type or Ascl1 mutant were plated onto uncoated tissue culture plastic with the addition of $2 \mu \mathrm{g} \mathrm{ml}^{-1}$ laminin (Sigma) to the medium (Euromed) supplemented with epidermal growth factor and fibroblast growth factor (both at $10 \mathrm{ng} \mathrm{ml}^{-1}$; Peprotech) incubated at $37^{\circ} \mathrm{C}$ under $5 \% \mathrm{CO}_{2}$ atmosphere. P19 and NS5 cells were plated in 24-well plates and transfected with Lipofectamine 2000 reagent according to the manufacture's protocol (Invitrogen). Twenty-four hours post transfection, cells were collected for protein or RNA extraction.

Ex vivo cortical electroporation. Ex vivo electroporation was performed on injected mouse embryo heads. The electrical parameters were: $50 \mathrm{~V}, 50 \mathrm{~ms}$ length, 5 pulses and $1 \mathrm{~s}$ interval. After electroporation, brains were dissected in Gey's buffer (Sigma) and cut coronally $(300 \mu \mathrm{m})$ with a tissue chopper (McIlwain). Slices were transferred onto sterilized culture plate inserts $(0.4-\mu \mathrm{m}$ pore size; Millicell-CM, Millipore) and cultivated in complete Neurobasal containing Neurobasal medium (Invitrogen) supplemented with 1\% B27, 1\% N2, 1\% glutamine, 1\% penicillin/ streptomycin and $1 \%$ fungizone. Slices were cultivated for 1 day followed by dissection of the GFP + portion of the cortex under a GFP binocular. Cells were dissociated in HBSS (Sigma) mechanically with round Pasteur pipettes, seeded on poly-Dlysine- $\left(10 \mu \mathrm{g} \mathrm{ml}^{-1}\right.$, Sigma $)$ and laminin $\left(10 \mu \mathrm{g} \mathrm{ml}^{-1}\right.$, Sigma)-coated wells (Lab-Tek, Chamber Slide, four-well Permanox slides) and cultured in complete Neurobasal medium for neuronal differentiation or cultured in proliferation medium (Euromed) supplemented with epidermal growth factor and fibroblast growth factor (both at $10 \mathrm{ng} \mathrm{ml}^{-1}$; Peprotech).

In situ hybridization. To determine the expression profile of Cenpj, we performed messenger RNA (mRNA) hybridization directly on tissue sections. Embryonic brains were fixed overnight in $4 \%$ paraformaldehyde (PFA) and transferred into $20 \%$ sucrose. After overnight incubation, the brains were embedded in optimal cutting temperature compound (OCT) (VWR International) and coronally cryosectioned $(12 \mu \mathrm{m})$. The sections were post-fixed with $4 \%$ PFA and treated with $0.25 \%$ acetic anhydride and triethanolamine to acetylate the amino groups on proteins and reduce the background. After a pre-hybridization step at $70^{\circ} \mathrm{C}$, the sections were incubated overnight at $70^{\circ} \mathrm{C}$ with digoxigenin-labelled RNA probes complementary to target mRNA. To detect the riboprobes that bound to the tissue, sections were incubated overnight at $4{ }^{\circ} \mathrm{C}$ with an anti-digoxigenin antibody conjugated with alkaline phosphatase. The signal was then revealed by adding nitro blue tetrazolium 5-bromo 4-chloro 3-indolyl phosphate (Roche), the substrate of alkaline phosphatase, to the sections.

In utero electroporation and tissue processing. In utero electroporation of the cerebral cortex was performed in anesthetized time-pregnant mice (14.5 days), as previously described ${ }^{6}$. Briefly, one telencephalic embryonic ventricle for each embryo was injected with $1 \mu \mathrm{l}$ of $1 \mu \mathrm{g}^{-1}$ endotoxin-free DNA. Cortices were electroporated with five 50-ms electrical pulses at $30 \mathrm{~V}$ with 1 -s intervals using 5 -mm platinum electrodes (Harvard Apparatus). At the desired time point after electroporation, mice were culled and embryos were processed for tissue analyses. Embryonic brains were fixed in 4\% PFA overnight and then placed in 20\% sucrose/ PBS overnight. Embryonic brains were then embedded in OCT and frozen before sectioning using a cryostat. After immunostaining, all images were acquired with a laser scanning confocal microscope (Leica SP5). Cell counts

were performed using Fiji (ImageJ). The different cortical zones namely VZ, SVZ, IZ and CP were identified based on cell density visualized with $4^{\prime}, 6$-diamidino-2phenylindole (Invitrogen) nuclear counterstaining. The CP was equally subdivided in three bins called uCP, medium CP and lower CP. Tuj1 neuronal marker staining co-localized with GFP was used to further identify newborn neurons in the VZ and SVZ, and migrating neurons in the IZ and CP.

Immunostaining. After washing in PBS, sections were treated with PBS-0.1\%, Triton X-100-10\% serum for $30 \mathrm{~min}$. They were then incubated overnight at $4{ }^{\circ} \mathrm{C}$ with the following primary antibodies diluted in the blocking buffer: mouse antiacetylated tubulin 1:100 (Sigma); mouse anti- $\alpha$-tubulin (1:100, Sigma-Aldrich); rabbit anti-Cenpj: 1:200 (Proteintech); rabbit anti-Cdk5rap2 (1:100, Milipore); rabbit anti-centrin: 1:100 (Milipore); chicken anti-GFP (1:700, Millipore); rabbit anti-pH3 (1:200, Upstate); mouse anti- $\gamma$-tubulin (1:100, Abcam) and mouse anti-tyrosinated tubulin 1:100 (Sigma). Sections were then incubated with appropriate fluorescent secondary antibodies. To detect Cenpj, a step of antigen retrieval with citrate was performed before the blocking (sodium citrate $\mathrm{pH}=6$ for $20 \mathrm{~min}$ at $\left.90^{\circ} \mathrm{C}\right)$.

Nocodazole treatment. After two days in vitro, cultures were treated for $30 \mathrm{~min}$ with $6 \mu \mathrm{M}$ nocodazole (Sigma) or dimethylsulphoxide (Sigma) and fixed with $4 \%$ formaldehyde at $37^{\circ} \mathrm{C}$ for $2 \mathrm{~min}$ followed by $3 \mathrm{~min} \mathrm{MeOH}$ at $-20^{\circ} \mathrm{C}$, as described previously ${ }^{31}$.

Quantification of immunohistochemical data. Immunofluorescence mean of acetylated or tyrosinated microtubules from the nucleus area was measured with ImageJ. Centrosome diameter was measured in each centrosome of the mitotic cell with ImageJ and the area was quantified $\left(\mu \mathrm{m}^{2}\right)$. Leading process thickness was measured by drawing a transversal line at the portion of the leading process adjacent to the nucleus with ImageJ. Mitotic spindle orientation and morphology was measured in high-magnification confocal images of cortical sections using ImageJ orthogonal three-dimensional viewer of hyperstacks. Taking into consideration the ventricle lining labelled with $\gamma$-tubulin and using the threedimensional viewer to ensure that the centrosome analysed belongs to the metaphase cell examined, we measured the angle between the cleavage plane and the ventricle. Immunofluorescence mean of the astral microtubule area was measured with ImageJ. Spindles were classified as bipolar or monopolar, symmetric or asymmetric, as described previously ${ }^{13}$

Plasmids constructs and siRNAs. Cenpj shRNA (5'-TCCGACTGGATGCCTG GAAGAGAGCAGAA- $3^{\prime}$ ), Ascl1 shRNA (5'-AGAAGATGAGCAAGGTGGAGA CGCTGCGC- $\left.3^{\prime}\right)$ control shRNA (5'-GCACTACCAGAGCTAACTCAGATAGT ACT- $\left.3^{\prime}\right)$ plasmids were purchased from Origene. Cenpj siRNA $\left(5^{\prime}\right.$-GGAGUAAA AUUGACUUCGA- $3^{\prime}$ ) and Silencer Negative Control No. 1 siRNA (catalogue number AM4636) were purchased from Ambion. To allow conditional expression of control shRNA and Cenpj shRNA using the Cre-LoxP system, sequences were cloned into pCALSL mir-30 vector (Addgene plasmid 13786), as previously described $^{54}$, and verified by western blots (Supplementary Fig. 3f). EB1-GFP plasmid was purchased from Addgene (plasmid 17234).

Real-time PCR. Cenpj mRNA expression was quantified by quantitative real-time PCR in control and shRNA-treated samples. RNAs were extracted from P19 cells or whole-telencephalon tissue using TRIzol reagent (Invitrogen), followed by a classical phenol/chloroform separation. The aqueous phase was mixed with isopropanol to precipitate the nucleic acid, which was then washed with $70 \%$ ethanol and resuspended in RNAse-free water. A clean-up step was then carried out using the RNeasy mini kit protocol (Qiagen). Purified RNAs were then reversetranscribed into cDNA using the Applied Biosystems kit. Real-time PCR was performed using Taqman probes according to the manufacturer's protocol (TaqMan gene expression assays, Applied Biosystems). The Taqman probes for Cenpj and for the endogenous control, $\beta$-actin, was purchased from Applied Biosystems. The 7500-system software provided by Applied Biosystem was used to run the PCR and analyse the data.

Statistical analysis. Data are expressed as mean \pm s.e.m. Statistical analysis was performed using an unpaired two-tailed Student's $t$-test with Prism software, version $6 .{ }^{\star} P<0.05,{ }^{* *} P<0.01,{ }^{* * *} P<0.001,{ }^{* * * *} P<0.0001$.

Western blotting. To determine the efficiency of the shRNAs, we analysed the expression levels of the protein of interest in control and shRNA-treated samples, by SDS-polyacrylamide gel electrophoresis protein separation and immunoblotting. Protein extracts from P19 cells or whole-telencephalon tissue were obtained by lysis with the Promega passive lysis buffer complemented with a cocktail of protease inhibitors (Roche). Western blotting was performed using standard protocols ${ }^{55}$. The primary antibodies were diluted in the blocking solution at the 
following concentrations: rabbit anti-Cenpj: 1:400 (Proteintech), rabbit anti-actin, 1:1,000 (Sigma, A2066) and rabbit anti-alpha tubulin, 1:1,000 (Sigma) and mouse anti-Mash1, 1:1,000, BD bioscience. Horseradish peroxidase-conjugated secondary antibodies were used as follows: goat anti-rabbit, 1:200 (Dako, P0449) and goat anti-mouse, 1:1,000 (Sigma, A8924). Uncropped scans of Fig. 1b and Supplementary Fig. 3f blots are shown in Supplementary Fig. 6.

\section{References}

1. Dobyns, W. B., Stratton, R. F. \& Greenberg, F. Syndromes with lissencephaly. I: Miller-Dieker and Norman-Roberts syndromes and isolated lissencephaly. Am. J. Med. Genet. 18, 509-526 (1984).

2. Norman, M. G., Roberts, M., Sirois, J. \& Tremblay, L. J. Lissencephaly. Can. J. Neurol. Sci. 3, 39-46 (1976).

3. Casarosa, S., Fode, C. \& Guillemot, F. Mash1 regulates neurogenesis in the ventral telencephalon. Development 126, 525-534 (1999).

4. Britz, O. et al. A role for proneural genes in the maturation of cortical progenitor cells. Cereb. Cortex 16 (Suppl 1), i138-i151 (2006)

5. Pacary, E., Azzarelli, R. \& Guillemot, F. Rnd 3 coordinates early steps of cortical neurogenesis through actin-dependent and -independent mechanisms. Nat. Commun. 4, 1635 (2013).

6. Pacary, E. et al. Proneural transcription factors regulate different steps of cortical neuron migration through Rnd-mediated inhibition of RhoA signaling. Neuron 69, 1069-1084 (2011).

7. Ge, W. et al. Coupling of cell migration with neurogenesis by proneural bHLH factors. Proc. Natl Acad. Sci. USA 103, 1319-1324 (2006).

8. Castro, D. S. et al. A novel function of the proneural factor Ascl1 in progenitor proliferation identified by genome-wide characterization of its targets. Genes Dev. 25, 930-945 (2011).

9. Leal, G. F. et al. A novel locus for autosomal recessive primary microcephaly (MCPH6) maps to 13q12.2. J. Med. Genet. 40, 540-542 (2003).

10. Gul, A. et al. A novel deletion mutation in CENPJ gene in a Pakistani family with autosomal recessive primary microcephaly. J. Hum. Genet. 51, 760-764 (2006).

11. Bond, J. et al. A centrosomal mechanism involving CDK5RAP2 and CENPJ controls brain size. Nat. Genet. 37, 353-355 (2005).

12. Al-Dosari, M. S., Shaheen, R., Colak, D. \& Alkuraya, F. S. Novel CENPJ mutation causes Seckel syndrome. J. Med. Genet. 47, 411-414 (2010).

13. Kitagawa, D. et al. Spindle positioning in human cells relies on proper centriole formation and on the microcephaly proteins CPAP and STIL. J. Cell Sci. 124, 3884-3893 (2011).

14. Tang, C. J., Fu, R. H., Wu, K. S., Hsu, W. B. \& Tang, T. K. CPAP is a cell-cycle regulated protein that controls centriole length. Nat. Cell Biol. 11, 825-831 (2009).

15. McIntyre, R. E. et al. Disruption of mouse Cenpj, a regulator of centriole biogenesis, phenocopies Seckel syndrome. PLoS Genet. 8, e1003022 (2012).

16. Bazzi, H. \& Anderson, K. V. Acentriolar mitosis activates a p53-dependent apoptosis pathway in the mouse embryo. Proc. Natl Acad. Sci. USA 111, E1491-E1500 (2014).

17. Sakai, D., Dixon, J., Dixon, M. J. \& Trainor, P. A. Mammalian neurogenesis requires Treacle-Plk1 for precise control of spindle orientation, mitotic progression, and maintenance of neural progenitor cells. PLoS Genet. 8, e1002566 (2012).

18. Konno, D. et al. Neuroepithelial progenitors undergo LGN-dependent planar divisions to maintain self-renewability during mammalian neurogenesis. Nat. Cell Biol. 10, 93-101 (2008).

19. Yingling, J. et al. Neuroepithelial stem cell proliferation requires LIS1 for precise spindle orientation and symmetric division. Cell 132, 474-486 (2008).

20. Feng, Y. \& Walsh, C. A. Mitotic spindle regulation by Ndel controls cerebral cortical size. Neuron 44, 279-293 (2004).

21. Goebbels, S. et al. Genetic targeting of principal neurons in neocortex and hippocampus of NEX-Cre mice. Genesis 44, 611-621 (2006).

22. Marin, O., Valdeolmillos, M. \& Moya, F. Neurons in motion: same principles for different shapes? Trends Neurosci. 29, 655-661 (2006).

23. Baudoin, J. P., Alvarez, C., Gaspar, P. \& Metin, C. Nocodazole-induced changes in microtubule dynamics impair the morphology and directionality of migrating medial ganglionic eminence cells. Dev. Neurosci. 30, 132-143 (2008).

24. Umeshima, H., Hirano, T. \& Kengaku, M. Microtubule-based nuclear movement occurs independently of centrosome positioning in migrating neurons. Proc. Natl Acad. Sci. USA 104, 16182-16187 (2007).

25. Valiente, M. \& Marin, O. Neuronal migration mechanisms in development and disease. Curr. Opin. Neurobiol. 20, 68-78 (2010).

26. Shim, S. Y. et al. Protein 600 is a microtubule/endoplasmic reticulumassociated protein in CNS neurons. J. Neurosci. 28, 3604-3614 (2008)

27. Tanaka, T. et al. Lis1 and doublecortin function with dynein to mediate coupling of the nucleus to the centrosome in neuronal migration. J. Cell Biol. 165, 709-721 (2004).

28. Hung, L. Y., Chen, H. L., Chang, C. W., Li, B. R. \& Tang, T. K. Identification of a novel microtubule-destabilizing motif in CPAP that binds to tubulin heterodimers and inhibits microtubule assembly. Mol. Biol. Cell 15, 2697-2706 (2004).

29. Hsu, W. B. et al. Functional characterization of the microtubule-binding and -destabilizing domains of CPAP and d-SAS-4. Exp. Cell Res. 314, 2591-2602 (2008).

30. Janke, C. \& Kneussel, M. Tubulin post-translational modifications: encoding functions on the neuronal microtubule cytoskeleton. Trends Neurosci. 33, 362-372 (2010).

31. de Anda, F. C., Meletis, K., Ge, X., Rei, D. \& Tsai, L. H. Centrosome motility is essential for initial axon formation in the neocortex. J. Neurosci. 30, 10391-10406 (2010).

32. Witte, H., Neukirchen, D. \& Bradke, F. Microtubule stabilization specifies initial neuronal polarization. J. Cell Biol. 180, 619-632 (2008).

33. Matov, A. et al. Analysis of microtubule dynamic instability using a plus-end growth marker. Nat. Methods 7, 761-768 (2010).

34. Hung, L. Y., Tang, C. J. \& Tang, T. K. Protein 4.1R-135 interacts with a novel centrosomal protein (CPAP) which is associated with the gamma-tubulin complex. Mol. Cell Biol. 20, 7813-7825 (2000).

35. Nieto, M., Schuurmans, C., Britz, O. \& Guillemot, F. Neural bHLH genes control the neuronal versus glial fate decision in cortical progenitors. Neuron 29, 401-413 (2001).

36. Parras, C. M. et al. Divergent functions of the proneural genes Mash1 and Ngn2 in the specification of neuronal subtype identity. Genes Dev. 16, 324-338 (2002).

37. Postiglione, M. P. et al. Mouse inscuteable induces apical-basal spindle orientation to facilitate intermediate progenitor generation in the developing neocortex. Neuron 72, 269-284 (2011).

38. Kirkham, M., Muller-Reichert, T., Oegema, K., Grill, S. \& Hyman, A. A. SAS-4 is a C. elegans centriolar protein that controls centrosome size. Cell 112, 575-587 (2003).

39. Chavali, P. L., Putz, M. \& Gergely, F. Small organelle, big responsibility: the role of centrosomes in development and disease. Philos. Trans. R. Soc. Lond. B. Biol. Sci. 369, 20130468-20130479 (2014).

40. Cormier, A. et al. The PN2-3 domain of centrosomal P4.1-associated protein implements a novel mechanism for tubulin sequestration. J. Biol. Chem. 284, 6909-6917 (2009).

41. Insolera, R., Bazzi, H., Shao, W., Anderson, K. V. \& Shi, S. H. Cortical neurogenesis in the absence of centrioles. Nat. Neurosci. 17, 1528-1535 (2014)

42. Toyo-Oka, K. et al. Recruitment of katanin p60 by phosphorylated NDEL1, an LIS1 interacting protein, is essential for mitotic cell division and neuronal migration. Hum. Mol. Genet. 14, 3113-3128 (2005).

43. Giampietro, C. et al. Stathmin expression modulates migratory properties of GN-11 neurons in vitro. Endocrinology 146, 1825-1834 (2005).

44. Westerlund, N. et al. Phosphorylation of SCG10/stathmin-2 determines multipolar stage exit and neuronal migration rate. Nat. Neurosci. 14, 305-313 (2011).

45. Buchman, J. J., Durak, O. \& Tsai, L. H. ASPM regulates Wnt signaling pathway activity in the developing brain. Genes Dev. 25, 1909-1914 (2011).

46. Fish, J. L., Kosodo, Y., Enard, W., Paabo, S. \& Huttner, W. B. Aspm specifically maintains symmetric proliferative divisions of neuroepithelial cells. Proc. Nat Acad. Sci. USA 103, 10438-10443 (2006).

47. Higgins, J. et al. Human ASPM participates in spindle organisation, spindle orientation and cytokinesis. BMC Cell Biol. 11, 85 (2010).

48. Fujimori, A. et al. Disruption of Aspm causes microcephaly with abnormal neuronal differentiation. Brain Dev. 36, 661-669 (2013).

49. Chansard, M., Hong, J. H., Park, Y. U., Park, S. K. \& Nguyen, M. D. Ndell, Nudel (Noodle): flexible in the cell? Cytoskeleton (Hoboken) 68, 540-554 (2011).

50. van der Voet, M. et al. NuMA-related LIN-5, ASPM-1, calmodulin and dynein promote meiotic spindle rotation independently of cortical LIN-5/GPR/Galpha. Nat. Cell Biol. 11, 269-277 (2009).

51. Rujano, M. A., Sanchez-Pulido, L., Pennetier, C., le Dez, G. \& Basto, R. The microcephaly protein Asp regulates neuroepithelium morphogenesis by controlling the spatial distribution of myosin II. Nat. Cell Biol. 15, 1294-1306 (2013).

52. Basto, R. et al. Flies without centrioles. Cell 125, 1375-1386 (2006).

53. Guillemot, F. et al. Mammalian achaete-scute homolog 1 is required for the early development of olfactory and autonomic neurons. Cell 75, 463-476 (1993).

54. Matsuda, T. \& Cepko, C. L. Controlled expression of transgenes introduced by in vivo electroporation. Proc. Natl Acad. Sci. USA 104, 1027-1032 (2007).

55. Azzarelli, R. et al. An antagonistic interaction between PlexinB2 and Rnd3 controls RhoA activity and cortical neuron migration. Nat. Commun. 5, 3405 (2014).

\section{Acknowledgements}

We gratefully acknowledge Drs Angeliki Achimastou, Debora Keller, Debbie Van den Berg, Emilie Pacary, Froylan Calderon de Anda, Hendrik Wilder, Isabelle Blomfield, Koji 
Oishi, Noelia Urban, Rebecca McIntyre and Roberta Azzarelli for advice on experiments or comments on the manuscript, and Elodie Ladeveze, Hayley Woods, Lan Chen, Vidya Ramesh, Wayne Turnbull and Wai Han Wong for technical support. We thank Dr Mary E. Hatten for providing the pClG2-Centrin2-Venus construct, Dr Pierre Gönzcy for the CPAP antibody and the constructs GFP-tagged full-length CPAP, GFP-tagged full-length CPAP deltaPN2-3 and GFP-tagged full-length CPAP deltaTCP. We thank Dr Tang K. Tang for the constructs shRNA11-centrinGFP and shcontrol-centrinGFP. P.P.G. was supported by a Medical Research Council Career Development Fellowship. This work was supported by a project grant from the Wellcome Trust $(086947 / \mathrm{Z} / 08 / \mathrm{Z})$ and by a Grant-in-Aid from the Medical Research Council (U117570528) to F.G.

\section{Author contributions}

P.P.G. and F.G. conceived all the experiments, analysed the data and wrote the manuscript. Together with P.P.G., J.D.-A. and I.C.-E. participated in carrying out experiments requested by reviewers. D.C. contributed with ChIP-chip data. D.B. helped with live imaging experiments and analysis of EB1-comets. All authors discussed the results and commented on the manuscript.

\section{Additional information}

Supplementary Information accompanies this paper at http://www.nature.com/ naturecommunications/

Competing financial interests: The authors declare no competing financial interests.

Reprints and permission information is available online at http://npg.nature.com/ reprintsandpermissions

How to cite this article: Garcez, P. P. et al. Cenpj/CPAP regulates progenitor divisions and neuronal migration in the cerebral cortex downstream of Ascl1. Nat. Commun. 6:6474 doi: 10.1038/ncomms7474 (2015).

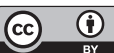
article are included in the article's Creative Commons license, unless indicated otherwise in the credit line; if the material is not included under the Creative Commons license, users will need to obtain permission from the license holder to reproduce the material. To view a copy of this license, visit http://creativecommons.org/licenses/by/4.0/ 